\title{
Numerical Simulation of Landing Gear Noise via Weakly Coupled CFD-CAA Calculations
}

\author{
Stéphane Redonnet ${ }^{1}$ \\ ONERA (French Aerospace Centre), BP 72 - 29 av Division Leclerc, Châtillon, 92322, France \\ Guilherme Cunha ${ }^{2}$ \\ Instituto Nacional de Pesquisas Espaciais (INPE), Cachoeira Paulista/SP, Brasil \\ Saloua Ben Khelii ${ }^{3}$ \\ ONERA (French Aerospace Centre), BP 72 - 29 av Division Leclerc, Châtillon, 92322, France
}

\begin{abstract}
The present work is relevant from the numerical prediction of aircraft noise via aeroacoustics hybrid methods. It is part of a more extensive effort, which final objective is the development of a robust and accurate CFD-CAA weak coupling methodology. Within this framework, we focus here on the so-called surface coupling approach, a technique that allows conducting weakly coupled CFD-CAA calculations. Such approach (which had been previously developed and validated on simpler cases) is here applied to a ralistic problem of aircraft noise, given by the acoustic emission of a nose landing gear in approach flight (a configuration that was addressed in the Airbus LAGooN program). For doing so, several preliminary tasks are first addressed, which are carefully handled and thoroughly detailed. Two CFD-CAA coupled calculations are then conducted, both being based on i) a same CFD dataset coming from an unsteady aerodynamic calculation (zonal DES), and ii) two distinct CAA calculations of different characteristics ; first, a CFD-CAA calculation is conducted for the so-called 'baseline' configuration (i.e. isolated gear within a free-field flow), so as to validate the coupling procedure, as well as to generate a reference solution for subsequent assessment of the mean flow effects induced by the experimental set-up. The validation of the coupling procedure is conducted via a direct comparison of the CFD-CAA results with either experimental or numerical (CFD, CFD-FWH) outputs obtained in the near-, mid, and/or far-field. With the view of assessing the mean flow effects, an alternative CFD-CAA calculation is then performed, which incorporates the realistic sheared jet flow characterizing the anechoic facility. This allows assessing the (partial) convection and refraction effects induced by such jet mean flow, which helps underscoring better the relevance of the present CFD-CAA hybrid approach when it comes to handle real-life noise problems.
\end{abstract}

\section{Introduction}

$\mathrm{T}$ HE noise environment around airports is a major concern in the world, with many local communities exposed to high levels of aircraft noise. Effective reduction of such 'noise pollution' represents an important environmental challenge throughout the foreseeable future. The noise signature of an aircraft includes two main contributions, namely, the engine noise component, which includes noise radiation from both turbomachinery (fan, turbine, combustion, etc.) and the jet sound emission, and the airframe (or aerodynamic) noise component attributed to the interaction between turbulent flow over the airframe and the adjacent solid structures such as wings, slats, flaps, landing gears, and cavities, etc. Although the engine noise accounts for a dominant portion of the overall aircraft noise during take-off, the airframe noise component becomes equally important during the approach for landing, when the engine thrust is considerably reduced. Therefore, in their effort to develop quieter airplanes, aircraft manufacturers need to design airframes that minimize the intensity of flow induced acoustic radiation to the far field. Consequently, the development of capabilities that offer both a deeper understanding and an accurate prediction of the physical phenomena related to aerodynamic noise

\footnotetext{
${ }^{1} \mathrm{PhD}$, Research Scientist, CFD and Aeroacoustics Department, Onera, Stephane.Redonnet@ onera.fr.

${ }^{2} \mathrm{PhD}$, Associate Professor, Laboratório de Combustão e Propulsão, INPE.

${ }^{3} \mathrm{PhD}$, Research Scientist, Applied Aerodynamic Department, Onera.
} 
generation and propagation mechanisms has became a priority for the aerospace research community. In particular, numerical simulations have emerged as a valuable complement to the more traditional experimental methods.

\section{Aerodynamic Noise Prediction, Hybrid Approaches and CFD-CAA Coupling}

\section{A. Aerodynamic Noise Prediction}

Prediction of aerodynamic noise is a complex problem that includes physical phenomena over a broad range of spatio-temporal scales. More precisely, the generation of acoustic disturbances is driven by turbulent structures of high amplitude and small space-time correlations, while the propagation of the acoustic disturbances is characterized by waves of low amplitudes and large space-time correlations. Although both phenomena are ruled by the same compressible Navier-Stokes equations, they cannot be easily predicted via a single calculation because the computational resources required to solve all of the relevant scales would be far too high. Therefore, to make the numerical approach tractable in a practical context, the overall aeroacoustic problem is decomposed into a set of coupled subproblems that focus on individual subregions of the overall spatial domain. Each subproblem has a specific range of amplitudes and physical scales that can be addressed using a numerical method that is customized to the dominant physics in that subregion. Methods involving a mix of techniques in this manner are classified as hybrid methods for aeroacoustic prediction.

\section{B. Hybrid Approaches}

In a general sense, aeroacoustic hybrid methods are comprised of three stages (see Fig. 1), which are respectively devoted to the 1) acoustic generation and near-field propagation (over regions of turbulent flow), 2) mid-field propagation (over regions where the acoustic amplitudes are linear but the mean flow is non-uniform), and 3) far-field propagation (over the majority of the distance from the source to the observer at ground level, wherein the mean flow is virtually uniform). The acoustic generation and early propagation (stage 1) can be simulated with a compressible unsteady CFD approach, whether it involves DNS (Direct

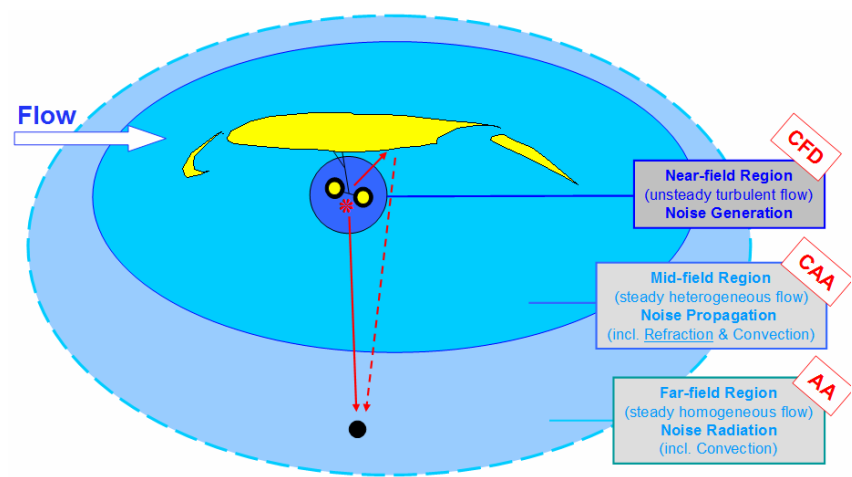

Figure 1. Hybrid Approach, for the airframe noise prediction

Numerical Simulation), LES (Large Eddy Simulation), unsteady Reynolds averaged Navier-Stokes equations, or a judicious mix of these techniques. The acoustic far-field radiation (stage 3 ) can be easily predicted with an Integral Method (IM), such as the well-known Kirchhoff or FWH (Ffowcs-Williams \& Hawkings ${ }^{1}$ ) integration techniques. The acoustic mid-field propagation (stage 2), can be neglected under certain special circumstances such as an isolated jet. However, the stage 2 cannot generally be ignored in airframe noise problems, because of the increased cost of extending the viscous, nonlinear CFD computations from stage 1 to include refraction across mean-flow non-uniformities and reflections by solid obstacles away from the region of acoustic generation. Although a Boundary Element Method (BEM) may be sufficient to model acoustic reflections, only higher fidelity computational aeroacoustics (CAA) approaches can simultaneously account for both reflection and refraction effects. Such high-fidelity CAA approaches must resolve the acoustic propagation over relatively large distances across a non-uniform mean flow, but do not need to account for turbulent fluctuations nor viscous effects on acoustic propagation. This may typically be accomplished with the Euler equations or a linearized version thereof.

\section{CFD-CAA (Weak) Coupling}

A critical aspect of developing such hybrid methodologies corresponds to the coupling, i.e., the information exchange, between the prediction modules for individual stages. The nature of this coupling is problem dependent, because of significant variations in the inter-dependencies between the various stages from one problem to another. However, except in problems involving acoustic feedback (e.g. screech tones), the coupling between the various stages is weak, i.e., primarily unidirectional. Under this scenario, feedback from a given stage to the previous one can then be neglected.

Implementing an appropriate one-way coupling from the unsteady CFD to the CAA stage is thus an essential ingredient in hybrid aeroacoustic methods that seek to simulate the full cycle of events associated with a complex aerodynamic noise problem, ranging from near-field generation to far-field radiation and including the mid-field propagation. As detailed in Ref. 2, it is not trivial at all to derive such a coupling, which has to 
ensure that all acoustic events generated in stage 1 as part of the CFD simulation will be correctly transmitted to the CAA solver for stage 2 in a conservative fashion (i.e. without any loss or duplication). At the present date, at least two approaches for coupling the CFD and CAA solvers exist, namely those based on 1) volumetric and 2) surface procedures, respectively.

D. CFD-CAA Surface (Weak) Coupling Technique Initially developed at Onera, the CFD-CAA surface coupling $^{3,4}$ (Redonnet et al., 2001) was successfully applied to several practical airframe noise applications $^{5-7}$, such as the acoustic emission by either an in-flight NACA0012 airfoil with a blunted trailing edge or a thick plate embedded within a flow.

More recently, the coupling technique itself was improved further (Redonnet, 2010) to deal with installed configurations ${ }^{2}$; more precisely, the coupling process was modified in order to allow a possible back scattering of acoustic waves through the coupling interface - a thing likely to occur whenever installed configurations are considered, since solid bodies surrounding the source region can reflect back anything the latter radiates.

This so-called Non Reflective Interfacing (NRI) technique ${ }^{2,8,16}$ was extensively used within a previous

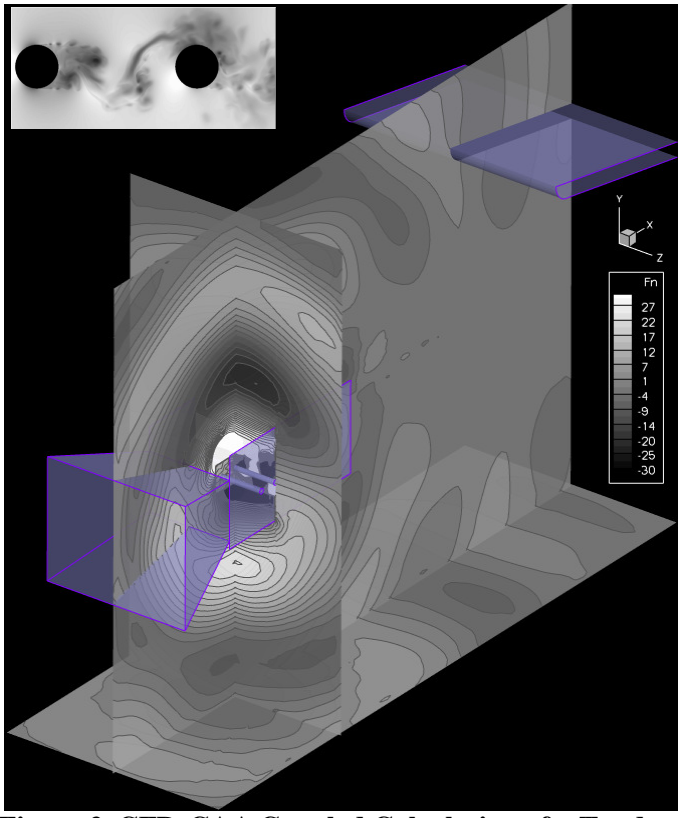

Figure 2. CFD-CAA Coupled Calculation of a Tandem Cylinder (TC), as installed within NASA/LaRC anechoic facility. Left corner: CFD calculation of the isolated TC (Lockard et al., NASA/LaRC). Main image: CFD-CAA coupled calculation, including the facility devices and jet effort, so as to enable CFD-CAA coupled calculations of the noise emitted by a Tandem Cylinder configuration including installation effects ${ }^{8}$ (see Fig. 2). Delivering both methodological and physical key insights, such work demonstrated further how far the CFD-CAA hybrid approach could effectively allow to improve the modelling of airframe noise problems, as well as to investigate the basic mechanisms underlying them.

\section{E. Optimization of the CFD-CAA Surface Coupling Procedure}

At that stage however, there was a need for optimizing the overall CFD-CAA surface coupling approach, in order to enable its application to realistic aircraft noise problems. In particular, it was needed to assess and improve the ways such hybrid methodology could 1) cope with all stringent constraints that are dictated by reallife applications, 2) without being jeopardized by some of their unavoidable side effects (such as the signal degradation to which CFD data are subjected, when manipulated for being then acoustically exploited ${ }^{14,27}$ ). With the view of answering those important and rather difficult questions, recently, several actions were conducted, which are summarized below (see Ref. 16 for more detail);

In a first time, theoretical studies were achieved in order to assess the possible signal degradation that CFD data can be subjected to, when sampled and/or interpolated in space and/or time. These works ${ }^{14,27}$ highlighted how far the acoustic information delivered by the CFD stage can be dramatically and irremediably degraded, depending on the way CFD data are effectively stored and/or processed for being acoustically extrapolated (whatever it is via a CAA approach, or via an IM technique - such as the FWH one). These investigations led to propose innovative solutions ${ }^{14,27}$ for better characterizing and possibly minimizing the impact that their manipulation can have onto CFD data. All these outcomes ${ }^{14,15,27}$ were extensively used in the present framework, allowing to handle at best the CFD data storage (and subsequent manipulation), as well as the CAA computational set up derivation.

In a second time, and for tentatively coping with all the inescapable constraints inherited from the signal degradation side effects previously evocated, several improvements were brought to the present CFD-CAA hybrid methodology; first, with the view of minimizing the sampling of CFD data to be stored (by decreasing at best their overall volume), the NRI (Non Reflective Interface) technique was optimized so that its minimal storage requirements could be relaxed. This was achieved by adapting the NRI procedure so that it may be handled by space operators of reduced stencil size, compared to those used for the propagation stage. This optimized version of the NRI, (which had been previously validated with the help of two academic test cases ${ }^{16}$ ) was used in the present framework, allowing to reduce by a factor two the amount of unsteady CFD data to be CAA-forced (which here, represented no less than 101 Gigabytes). Second, with the view of reducing at maximum the CAA grid density (and, thus, volume) to be handled for the propagation stage, a new class of optimized finite difference (FD) propagation schemes were developed (Cunha \& Redonnet, 2012); these so- 
called intrinsically optimized finite differences (IOFD) schemes ${ }^{24,27}$ are indeed of optimal accuracy, thanks to an optimization process that is based on a minimization of the scheme's leading-order truncation error (rather than on an optimization of the scheme' spectral properties, such as usually done ${ }^{25,26}$ ). Thanks to their optimal accuracy properties, these IOFD schemes allow dealing with much coarser CAA grids. More precisely, with no more than 4 Points Per Wavelength (PPW), an IOFD scheme of 15-point stencil guarantees that the error made on the group velocity (from which depends the acoustic energy transport and, thus, the overall accuracy of the CAA stage) is less than $0.1 \%$. Compared to the accuracy of a classical 7 -point stencil $/ 6^{\text {th }}$ order standard finite difference scheme $e^{25,26}$ (which corresponding minimal PPW is 12), this represents a gain of 3 per direction, i.e. a factor 27 in 3D. Needless to say, such benefit can be directly translated into a reduction (resp. an increase) of the CAA computational effort to be paid (resp. the frequency range to be reached), with memory requirements that go decreasing by the same factor, while CPU times can be reduced even more (depending on the CFL constraints). As a corollary, with respect to the present CFD-CAA coupling concerns, these reductions in the CPU/MEM needs by the CAA stage can be directly turned into a beneficial increase of the CFD storage grid which, at the present date, constitutes the best way for minimizing the signal degradation induced by sampling and/or interpolation operations ${ }^{14,27}$. Here again, the IOFD schemes (which had been previously validated via test cases of increasing complexity ${ }^{24,27}$ ) were applied to the present study. In particular, the latter was numerically handled via a 15-point IOFD scheme, allowing then to deal with a much lighter CAA grid volume (and, thus, calculation CPU time, etc), compared to the one(s) that would have been required if a regular 7-point $/ 6^{\text {th }}$ order standard FD scheme had been used, instead.

\section{Aeroacoustics of a Nose Landing Gear via a Hybrid Approach}

Once it had been optimized thanks to the various improvement recalled above, the CFD-CAA coupling procedure seemed mature enough for being tentatively applied to a realistic problem of industrial-like nature, such as the one given by the aeroacoustic emission of a nose landing gear (NLG). This is what motivated the present study, and constitutes the subject of this paper.

\section{A. Aeroacoustics of a Nose Landing Gear, via a CFD-FWH Hybrid Approach}

To better predict and assess the physical mechanisms associated with landing gear noise sources, a dedicated research program was initiated in 2007; funded by Airbus, the so-called LAGOON project (LAnding Gear NOise database for CAA validatiON) involved several partners, among which Onera, DLR, and Southampton University. Objective of the project was to acquire an extensive experimental database associated with an elementary configuration of a simplified nose landing gear (see Fig. 3), so that numerical tools dedicated to landing gear noise predictions can be accurately validated.

Within this framework, combined experimental and computational campaigns were thus carried out, focusing on both the aerodynamics and the acoustics of such simplified landing gear configuration. The model geometry was that of a nose gear of an Airbus A320 aircraft, with a scale factor of 1:2.5, and with

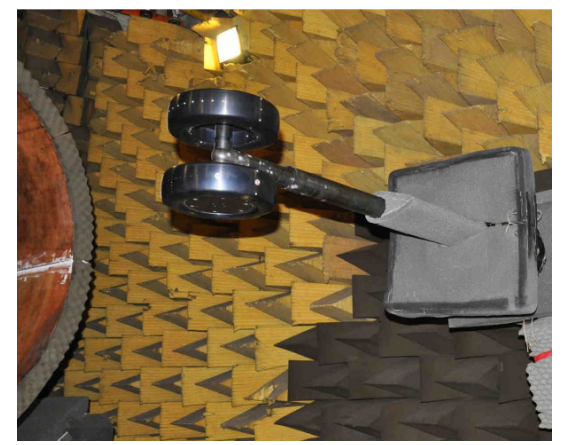

Figure 3. LAGooN Nose Langing Gear as installed in Onera's anechoic facility CEPRA19

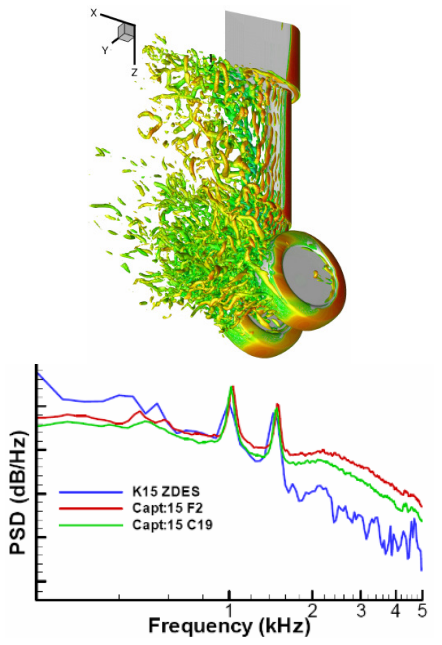

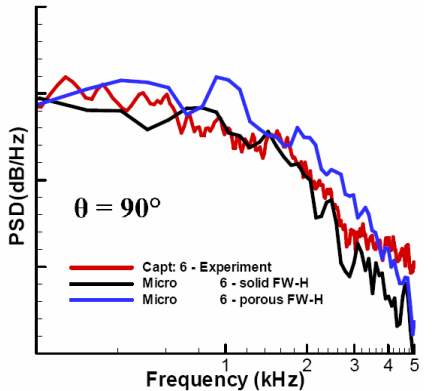

Figure 4. Aeroacoustics of a Simplified Nose Landing Gear via a CFD-

FWH Hybrid Approach. Near-field aerodynamic (left) and far-field acoustic (bottom) data. Left: CFD computation (top: $Q$-criterion, bottom, in blue: spectra of a wheel's probe, compared against experiments - in red and green).

Top: CFD-FWH hybrid calculation (black: solid surface, blue: porous surface) compared against experiments (red), for two far-field microphones located in the flyover (left) and side line (right) directions 
only the main elements (leg, wheels, etc) kept. The aero+acoustic dual experiments were achieved in Onera's aerodynamic $(F 2)$ and anechoic (CEPRA 19) facilities, respectively. The $14^{\text {th }}$ and $15^{\text {th }}$ AIAA-CEAS Aeroacoustic Conference provided an opportunity to thoroughly detail the experiments and associated results ${ }^{9,10}$.

The computational counterpart of such aero+acoustic experimental campaign was conducted at Onera; aerodynamics computations ${ }^{11}$ relied on 3D unsteady CFD calculations (based on the zDES approach), which were all conducted thanks to Onera's solver named elsA (see left side of Fig. 4, top). Those calculations were favourably compared with the aerodynamic experiments, through direct comparison of near-field results (see left side Fig. 4, bottom). In particular, both experimental and numerical outputs exhibited tonal noises (or approx $1 \mathrm{kHz}$ and $1.5 \mathrm{kHz}$ ), which emission was inferred to be associated with resonances coming from the wheels' inner cavities $^{9-12}$. The unsteady CFD results were then post-processed in an acoustic sense ${ }^{12,13}$, this being made with the help of a Ffowcs-Williams and Hawkings (FWH) approach and solver (Onera's code KIM). These CFDFWH coupled calculation results were also favourably compared to the experimental measurements recorded in the far-field (see right side of Fig. 4).

\section{B. Towards an Application of the CFD-CAA Hybrid Approach to the NLG Configuration}

Despite of the good match between numerical and experimental outputs, there was both a need and an interest in trying to go further in such an a posteriori assessment of NLG aeroacoustics, by tentatively improving the fidelity of the acoustic propagation stage;

First, it seemed necessary to account for the acoustic emission that had been effectively predicted by the CFD stage, rather than to model it via equivalent sources, as implicitly done in the FWH approach. Second, it appeared important of accounting for the realistic jet flow (and thus convection/refraction effects) characterizing the experiment, rather than to model it via a simplistic (uniform) mean flow - as also done in the FWH approach. These two requirements could be fulfilled by a CFD-CAA hybrid method such as the one previously introduced, provided however the latter could cope with all the stringent constraints and subsequent approximations such a realistic configuration could lead to.

Far beyond its sole applicative concerns, another indirect objective of the present effort was thus also to first answer more methodological questions, in order to establish and deliver general guidelines about how to properly handle CFD-CAA coupled calculations in non trivial situations, in particular regarding all constraints inherited from both fundamental and practical aspects (CFD data signal preservation ${ }^{15}$, CFD-CAA coupling feasibility ${ }^{2,8}$, CAA calculation stability and accuracy ${ }^{2,8,15,24}$, etc.). These methodological points were more particularly investigated and detailed in a previous work ${ }^{15,16}$, which delivered early results of a preliminary CFDCAA calculation ${ }^{16}$ of the present NLG configuration.

Once these questions had been answered, it became possible to make an effective use of the proposed methodology, for 1) assessing further and possibly validating the ability of the optimized CFD-CAA hybrid approach introduced above to effectively handle such a realistic configuration, as well as 2) possibly characterizing better its associated acoustic radiation. With that view, two CFD-CAA coupled calculations of the present NLG configuration were conducted, both being 1) performed using the CFD-CAA surface coupling procedure, and 2) based on the same CFD dataset (associated with the particular LAGooN configuration that addressed a flow stream of Mach and Reynolds number $M=0.18$ and $\operatorname{Re}_{D}=1.210^{6}$, respectively - see Ref. 13 for more detail). These CFD-CAA calculations are detailed and discussed hereafter.

\section{CFD-CAA Coupled Calculations of the NLG: Methodology and Computational Set-Up}

\section{A. Preliminary Tasks}

Before any CFD-CAA weakly coupled calculation could effectively be achieved, several preliminary (though sometimes intensive) tasks were conducted, which are summarized below (see Ref. 16 for more details).

\section{Specifications of the CFD storage to be achieved in space}

The choice of the source regions to better consider for applying the CFD-CAA coupling was based on the calculation results that had been previously obtained via a CFD-FWH hybrid approach ${ }^{11,12}$. Indeed such calculations had revealed that the NLG overall acoustic radiation was to be mainly dominated by the contribution coming from the wheel part, especially in the flyover direction. In addition, for saving computational resources, the CFD mesh had been slowly coarsened along the leg's region, which ending part exhibited then a 4 times lower density, compared to the one of the wheel's area; one could thus expect the CFD unsteady perturbations of the leg's zone to have been more importantly filtered out by the ( $2^{\text {nd }}$ order $)$ schemes, compared to that of the wheel's area. Finally, acquiring data over both the wheel and the leg regions would have led to a prohibitive cost in terms of amount of data to be stored. For all these various reasons, choice was here made of ignoring the leg's contribution, for focusing only on the wheel one.

Regarding now the area where to preferably prescribe the CFD-CAA coupling interface, choice was also made of locating it approximately at the same location than the one that had been initially specified for these 
prior FWH exploitations. Indeed, one can here remind that, as it is the case for CFD-FWH calculations, the CFD-CAA coupling interface has to be located as close to the source region as possible, without getting too much into regions of strong hydrodynamic activity (all this resulting from a compromise to be found between the accuracy of the unsteady aerodynamics stage and the stability of the acoustic one $)^{2}$. In the present case, locating properly the coupling interface was uneasy to achieve since it had to be done a priori, i.e. without any precise knowledge of the aero/acoustic field to be expected from the CFD stage. However, a rough analysis of the CFD material had indicated that, over the CFD-FWH coupling region, the cut-off frequency to be expected from the CFD grid was of approximately $3 \mathrm{kHz}$ (considering a minimum of 40 CFD Points Per Wavelength, with respect to the $2^{\text {nd }}$ order method used for the CFD stage). Since experimental and numerical results previously obtained for the slightly higher Mach number had indicated that tonal emissions were to occur below $2 \mathrm{kHz}$, such a $3 \mathrm{kHz}$ upper frequency limit seemed acceptable. On another hand, due to the rapid stretching of the CFD grid beyond this region, it would have not really been possible to locate the interface further away from this location. Finally, coming in addition to the accuracy/stability reasons evocated above, it appeared that locating the CFD-CAA interface in the vicinity of the CFD-FWH one could also help in minimizing the dissimilarities between the CAA and the FWH stages, so that the CAA vs. FWH comparisons to be conducted were biased at minima ${ }^{8}$.

Regarding now the exact design of the CFD data storage interface, choice was deliberately made of imposing to the latter a grid point density of similar characteristics than the one characterizing the CFD grid over the coupling area. This was indeed done with the view of preserving at best the signal to be stored, by preventing it from being degraded too much by all possible sampling and / or interpolations to be conducted in space (see Ref. 13-15, for further explanations). For a certain number of reasons (see Ref. 16 for more details), rather than deriving the CFD-CAA interface directly from the CFD mesh, choice was made of constituting it with the help of several slices of points coming from a CAA mesh of ideal characteristics, i.e. a Cartesian grid. Such an option offered the advantage of allowing to build a CAA mesh presenting both an easier handling (topology, etc.) and an optimal propagative behaviour (accuracy, stability, etc), all this thanks to its Cartesian nature. On another hand, such an alternative implied to space-interpolate the source signal from the CFD grid

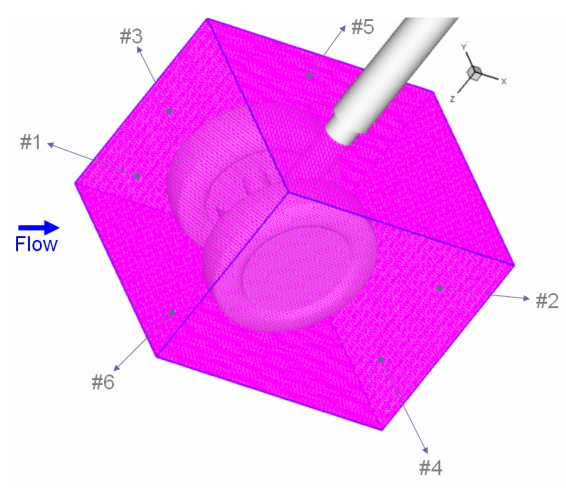

Figure 5. CFD-CAA Coupling Surface over the wheel region, composed of 6 interfaces gathering each 5 slices of cells towards the CAA one, with - as a possible result - a degradation of its content. In order to carefully investigate such point, a dedicated analysis was conducted (see Ref. 13, for details), which conclusions confirmed the relevance of the choice made. Consequently, all CFD data were stored over a Cartesian envelope (see Fig. 5), the latter being constituted of approx 800000 points, which were more or less equally distributed over 6 interfaces of 5 layers thickness.

\section{Specifications of the CFD storage to be achieved in time}

The choice of the rate with which storing the CFD data in time was also based on the computational set-up that had been previously used for the CFD-FWH coupled calculations ${ }^{11,12}$. Indeed, due to memory requirements, CFD storages are generally conducted along with a sampling in time (and sometimes in space) ${ }^{14,15,27}$. Such sampling in time is achieved at a rate that is usually determined a priori, based on a balance between several aspects, such as the minimization of the storage volume, the preservation of the acoustic information, the stability of the CAA calculation, etc. The fact is that it is especially in such a sampling operation that lies the aliasing phenomenon ${ }^{14,15,27}$, which may strongly degrade a signal, when sampled - a thing that may then jeopardize both the accuracy of the CFD-CAA source signal and the stability of its CAA-exploitation. However, identifying accurately the optimal sampling rate with which storing a given signal implies to analyze thoroughly the latter. In the present case, this would have required to first gather over several probes the totality of the CFD signal as is (i.e. without any sampling), which had not been done during the CFD computational campaign. Therefore, in the absence of more insights on that point, and considering that storing more data would have led to a prohibitive storage volume, choice was here made of prescribing a sampling rate of same value (1:10) than the one used for the FWH stage. The data were stored during the second half of the CFD calculation, leading to a total of 14,400 temporal occurrences (corresponding to a physical duration of $72 \mathrm{~ms}$ ) and an overall volume of 0.75 TeraBytes.

\section{Analysis of the CFD stored signal}

With the view of deriving a CAA grid that would not corrupt it too much, the stored (and possibly partly degraded) signal was then analyzed carefully. For doing so, use was made of Onera's cAmilA solver (Cunha, 2011), a tool that was developed especially for such CFD-CAA coupling purposes ${ }^{14,27}$; 
First, by examining the CFD perturbed signals that had been acquired over each storage interface encompassing the source zone (see Fig. 5), it was observed that part or totality of interfaces \#2 and \#5 (located downstream and above the wheels, respectively) exhibited very high amplitude levels, this being due to their crossing by hydrodynamic occurrences (vortices, etc.) convected by the wake coming from the NLG leg and /or wheels (see Fig. 7). In order to prevent the CAA calculation from being jeopardized by such hydrodynamic occurrences $^{2,8}$, these 2 interfaces were dropped down, and not considered any further for the CAA consumption. The 4 other interfaces presented far less important amplitudes,
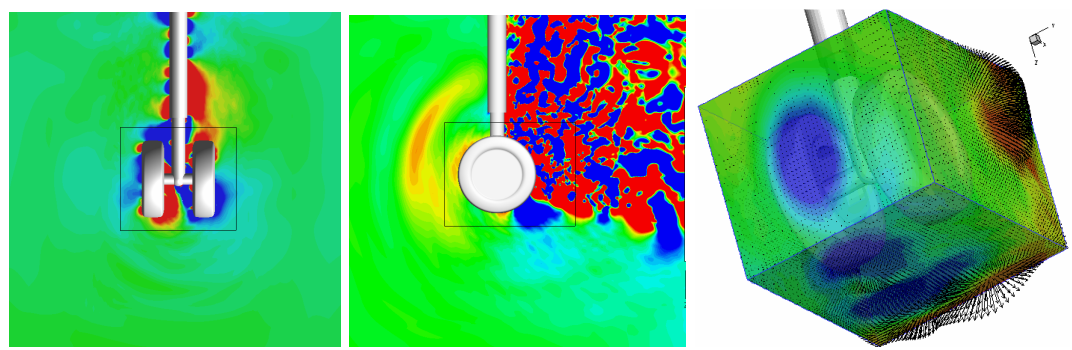

Figure 7. CFD Calculation. Left and center: unsteady data (perturbed pressure) computed and stored at approx. $1 / 3^{\text {rd }}$ of the storage time (black square indicates the storage interface). Right side; unsteady data plotted over the storage interface at approx. $2 / 3^{\text {rd }}$ of the storage time (black arrows depict the perturbed velocity vectors)

translating the fact that they were mainly crossed by acoustic waves - at least over their central part. Indeed, a careful observation of all instantaneous flow field realizations revealed that, at some moments, the downstream parts of the remaining interfaces were also impinged by unsteady aerodynamic occurrences of rather high intensity (see right side of Fig. 7). Apart from that, the analysis of the time signatures associated with the stored signal data revealed that, although the storage had been started only at mid-term of the CFD calculation, the latter was still not fully converged at that time. In particular, a transient state could still be observed during the first 2000 iterations of storage. The latter were thus dropped down, and not considered any further for the CAAexploitation.

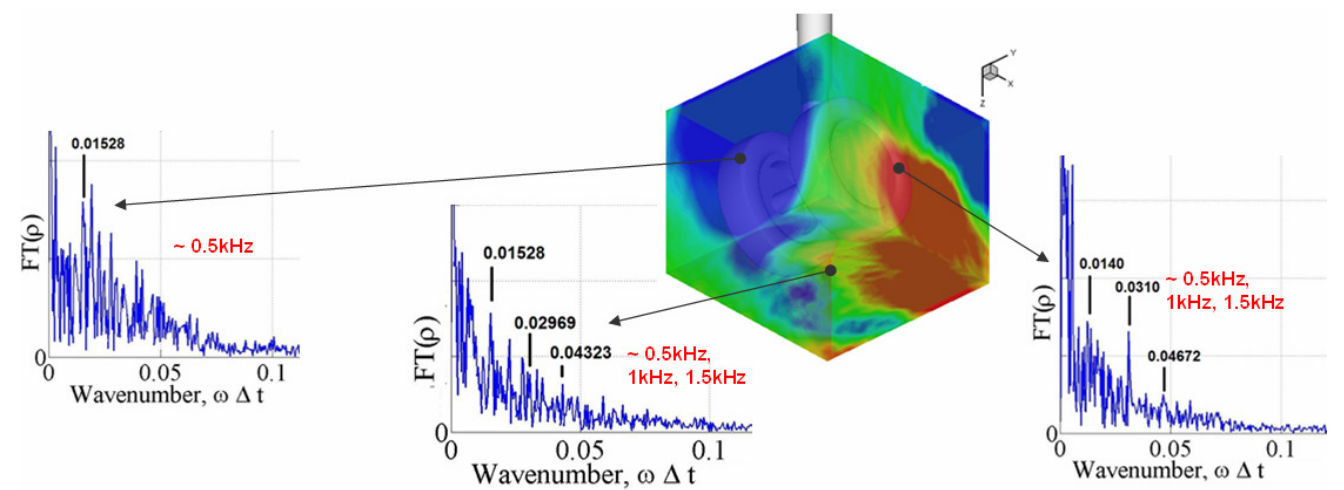

Figure 8. Frequency Spectra of the CFD Signal stored at the center of storage interfaces \#1, \#4, \#6 (from left to right)

Once its spectra was properly extracted with the help of Fourier transforms, the source signal revealed additional insights (see Fig. 8); first, it appeared that the CFD dataset had effectively gathered some of the tonal contents that were to be expected. Among others, tonal emissions of $500 \mathrm{~Hz}, 1 \mathrm{kHz}, 1.5 \mathrm{kHz}$ and sometimes $2 \mathrm{kHz}$ clearly emerged, depending on the interface that was considered. In addition, further analysis of these spectra led to the conclusion that, above a dimensionless frequency of $\omega \Delta_{\mathrm{t}_{\text {storage }}}=0.1$ (corresponding here to $f=3 \mathrm{kHz}$ ), the signal had certainly lost most of its physical meaning, exhibiting mainly corrupted (degraded and/or corrupted) information. This was indeed coherent with the expected cut-off frequency of $f=3 \mathrm{kHz}$ that had been estimated for the CFD grid. Below such a $3 \mathrm{kHz}$ limit, however, one could expect the signal to be more physical (and, thus, numerically reliable), although possibly gathering also some spurious contents (of low- to mid- frequency) inherited from the back-aliasing of originally high frequency modes, due to their sampling in time. In the absence of more knowledge on that matter, such a $3 \mathrm{kHz}$ limit was taken as the upper 'reliable' frequency limit, from which all criteria for effectively constructing the CAA grid was then be derived.

\section{Derivation of the CAA Grid}

The CAA grid was then constructed, following several criteria; first, such grid had to guarantee a correct propagation of the source signal over the frequency range of interest, while possibly allowing a proper capture of the convection and refraction effects by the mean flow. For doing so, and considering the intrinsically optimized schemes $^{26,27}$ of 15 points that were to be used here, a minimum of 4 Points Per Apparent Wavelength (PPW) was 
ensured over the whole domain. In addition, the CAA grid density was locally and progressively refined over all regions where a higher spatial resolution was required, such as the areas where the coupling interface was located (this, in order to avoid any space-aliasing of the source signal, when projected in the CAA domain).

Second, the CAA mesh had to extend up to the regions of interest (e.g. the areas where acoustic data had been experimentally recorded), while remaining small enough for ensuring that the computation and restitution (e.g. visualization) efforts could be kept reasonable enough (either in CPU time or memory). Since reaching all of the far field microphones was deemed to be impractical, the CAA domain was limited in extent, such that it corresponded to a cubic box of dimensions $[-15 R, 25 R] \times[-25 R, 25 \mathrm{R}] \times[-5 \mathrm{R}, 43 \mathrm{R}]$; as is, such CAA domain encompassed some of the microphones that had been used in the experiments, and that were located either in the far-field (in the flyover direction, at a distance of approx. $40 R$ from the gear axle - with $R$ indicating the wheels radius), or in the midfield (at approx. 13R, in the sideline and flyover directions).

Finally, as was said previously, the CAA mesh was built so that it extended naturally the CFD storage interface, with grid points matching exactly the CFD ones. On another hand, due to the fact that such CFD-CAA interface had naturally inherited from the CFD mesh density, it would have been prohibitive to keep all grid points in the CAA grid. Choice was here thus made of partially coarsening such CFD-CAA interface, by sampling it along its lateral directions, while keeping it as is in its normal direction. On that stage, two options were examined and numerically investigated; the lighter - but riskier - option consisted in prescribing a lateral sampling of 3, allowing then to minimize the CAA grid density. This option rose however a non negligible risk of leading the CFD source signal to be space aliased, when projected on the CAA grid (this, with respect to the maximal frequency and minimal cell size here considered). A safer - but heavier - option consisted in prescribing a lateral sampling of 2, a rate ensuring that less spatial aliasing would occur during the projection of the source signal onto the CAA grid. While the first option (1:3 sampling) had been retained for achieving preliminary calculations (which were conducted with the help of 11-points IOFD schemes, and led to mitigate results - see Ref. 16 for more detail), in the present study, the second option (1:2 sampling) was privileged. On another hand, the extra cost to be induced by such denser coupling interfaces (and, thus, CAA grid) was balanced by the use of 15-points IOFD schemes (which require a lighter mesh, compared to their counterpart of 11 points).

As was said, along the interfaces' normal direction, the CAA mesh density corresponded to the CFD storage interface one. Therefore, over the coupling region, the CAA grid was approx. 5 times denser than within the rest of the computational domain. The matching between these two regions was achieved thanks to a progressive stretching of the cells size, which rate was kept small enough (less than 3 per cent) for avoiding any back scattering of the propagated signal, due to grid cells heterogeneities ${ }^{14,15}$.

Finally, as had been done in previous studies devoted to academic test cases ${ }^{8,16}$, choice was here made of not CAA-meshing the NLG geometry. This indeed would have increased the amount of work/computational price to be done/paid, without bringing any substantial extra information (especially considering the relatively small dimensions of the gear). Over the source region, the CAA grid was thus kept as Cartesian, and the obstacle ignored.

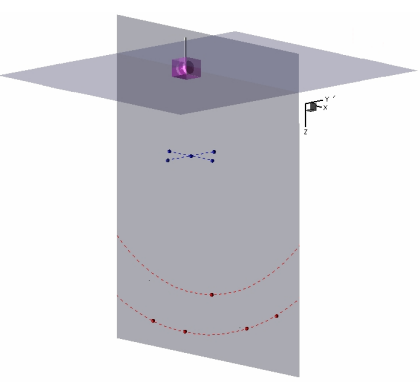

Figure 9. CAA Grid, of Cartesian heterogeneous nature (NLG is not meshed). CFD-CAA coupling surface drawn in purple. Mid- and far-field microphones (flyover direction) plotted as blue and red points, respectively

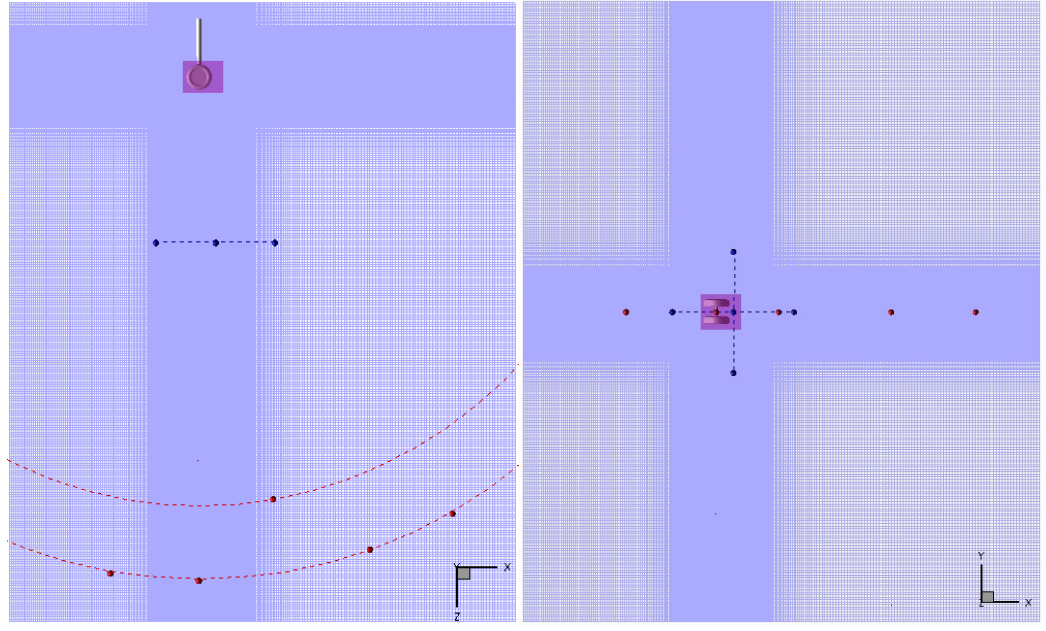

At the end, a CAA grid of 36 blocks and 66 million of points was derived via analytical means, being built up directly from the CFD storage interfaces (see Fig. 9). One can here recall that such grid was designed for matching the 4 PPW constraints of the intrinsically optimized 15 points schemes. Needless to say, if such grid had been designed for a 7-point standard FD scheme $e^{25,26}$, it would have led to a prohibitive number of grid cells (more than 1.5 billion of points). 


\section{Derivation of the CAA Steady Mean Flow}

Following the strategy adopted in a previous effort ${ }^{8}$, two different steady mean flows were considered for the CAA consumption;

First, an as-like 'free-field' homogeneous mean flow was derived, by simply prescribing at every CAA point the thermodynamic values of the flow at infinity (i.e. a free stream Mach number of 0.18). Such a uniform mean flow aimed at easing direct comparisons of the CFD-CAA coupled calculation outputs against either experimental data (which had been corrected from the flow effects by the facility jet ${ }^{28}$ ) or numerical CFD-FWH ones (which had been obtained under the same 'homogenous medium' assumption, due to the intrinsic limitation of the FWH approach). In addition, such a free-field homogeneous mean flow also aimed at providing a reference solution against which comparing results associated with a more realistic jet flow, allowing then to possibly assess the partial convection / refraction effects that might be induced by the jet shear layers characterizing the experimental set-up (see Fig. 3).

Indeed, in reality, the jet flow is initially confined within the nozzle walls. As the jet exits the nozzle, it spreads out itself in the ambient medium, this leading to the creation of shear layers which strength go decreasing with the axial distance. Needless to say, the refraction effects to be possibly induced by such shear layers may affect the acoustic emission, deflecting acoustic waves and modifying their associated directivity patterns. On the same way, the absence of any convection outside the region where the jet exists may lead to propagation patterns that differ from the ones associated with the free-field jet flow. In order to mimic at best such a sheared / confined character of the jet, and in the absence of any other data, an alternative mean flow was thus derived from the previous one. This was done following the approach used in a previous effort ${ }^{8}$, i.e. by modifying the free-field flow via analytical means, its aerodynamics primitive quantities being altered with the help of similarity functions. These functions were designed for spatially evolving, with an evolution rate driven by both 1) the axial distance to the nozzle exit plane and 2) the radial distance to the nozzle lips. The jet spreading out factor was calibrated for matching the theoretical growth factor of the turbulent boundary layer thickness, delivering a spreading angle (of 4 degrees) corresponding to ones recorded for the current installation (Onera's CEPRA 19 wind tunnel, in its a ' $2 m$ nozzle diameter' configuration). As an illustration, Figure 10 displays some views of the resulting mean flow associated with such an as-like facility-installed NLG configuration.
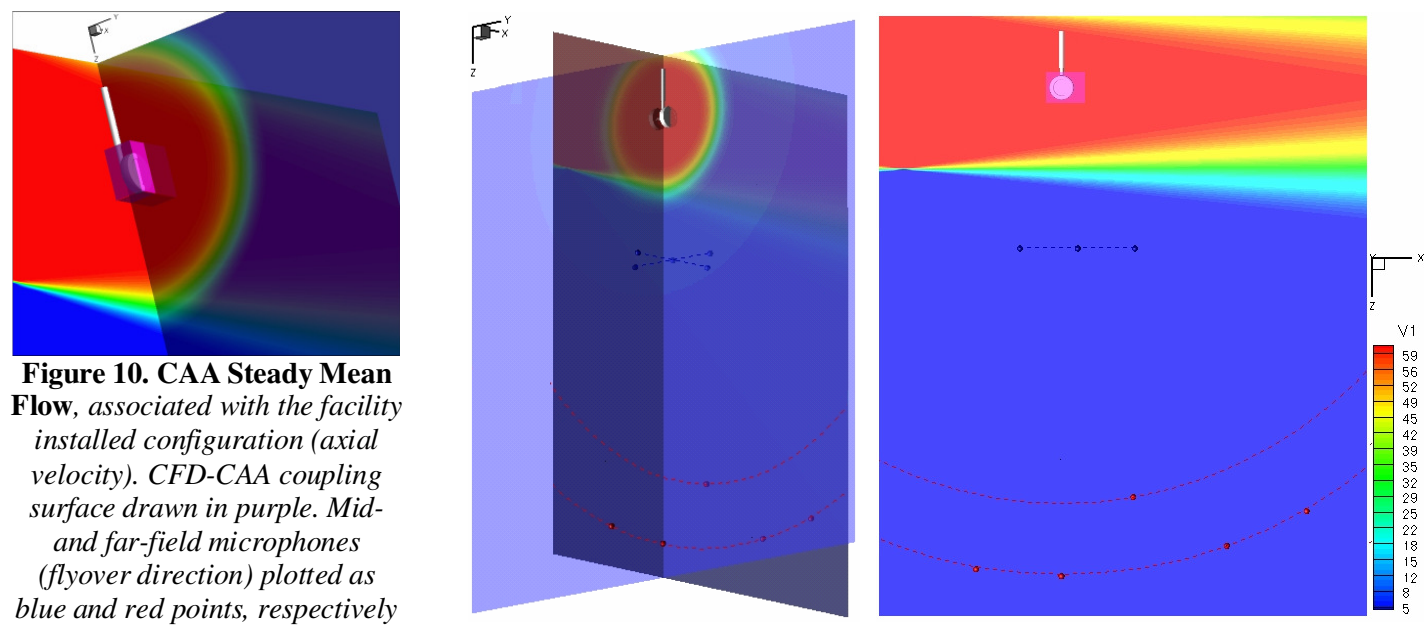

On that stage, one can notice that, contrarily to what had been done in a previous effort ${ }^{8}$ and although this would have allowed meeting a higher degree of realism, none of the facility devices (nozzle, collector, etc.) were considered here, since meshing and computing the entire configuration with all the experimental set-up incorporated would have led to prohibitive CPU costs.

\section{Derivation of the CAA Source Signal}

Finally, the perturbed source signal to be injected within the CAA stage was derived from the CFD dataset that had been previously stored. Although such a task could have been achieved in a straightforward manner by simply subtracting from the stored CFD total field its steady mean flow counterpart, here, choice was deliberately made of re-generating the perturbed source signal in a slightly different manner. Indeed, there is a legitimate concern of preferably forcing the CAA stage with fluctuating quantities of null average value (i.e. quantities oscillating effectively around zero), this in order to avoid any artificial drift in the source signal amplitude. On another hand, it is not rare that unsteady CFD calculations are not fully converged, delivering instantaneous quantities that drift slowly over the time, and so their averaged value. In order to rigorously take 
that drift into account, it would then be needed to derive the perturbed quantities from their total counterpart by subtracting from the latter an alternative mean flow, of time dependent nature - which may not be so easy to determine. Another and easier option consists in considering only that portion of the CFD calculation which is fully converged, i.e. that part of the stored data which effectively presents a time-independent average value. As was said before, in the present case, the stored signal had been cleaned from its transient state (by dropping down its 2000 first iterations); the perturbed source signal was thus easily derived from its total counterpart, by subtracting from the latter its effective mean value - which was obtained by time averaging only the temporal records effectively kept.

Finally, regardless of whether the steady mean flow was considered as a 'free-field' or 'facility installed' one, the same perturbed source signal was considered for being injected into the CAA calculation. This was done so that the acoustic source could be free of any bias possibly induced by different mean / perturbed splitting definitions. Furthermore, this was justified by theoretical considerations which indicate that, as long as a perturbation problem remains linear, the fluctuating field is independent of the mean state (except for refraction effects).

\section{B. Computational Set Up}

As was said, two CFD-CAA weakly coupled calculations were conducted, all being based on the almost same computational set up, and fed with the same CFD dataset.

The CAA stage was conducted with the help of ONERA's sAbrinA.vO solver ${ }^{3,4,7,17-19}$ (Redonnet et al, since 2001). One can here remind that $s A b r i n A . v O$ is a structured, time-accurate CAA code that solves either the full or the linear Euler equations, in a conservative and perturbed form (with a splitting of the complete variables into a 'frozen' mean flow and a 'fluctuating' perturbation). The solver employs high-order, finite-difference operators, involving $6^{\text {th }}$-order spatial derivatives and $10^{\text {th }}$-order filters, as well as a $3^{\text {rd }}$-order, multi-stage, Runge-Kutta timemarching scheme. The code deals with multi-block structured grids with one-to-one interfaces, and is fully parallelized using the Message Passing Interface (MPI) standard. Finally, the solver includes the usual boundary conditions (reflection by solid walls, non-reflecting / free-field radiation ${ }^{3,4,6}$, etc.), as well some unique to specific applications (such as the Non Reflective Interface technique ${ }^{2,8}$ that was extensively used in the present effort). More detailed information about the sAbrinA. $v 0$ solver and its underlying methodology can be found in Refs. ${ }^{3,4,7,17-19}$.

As was said previously, in the present effort, the 7-point standard FD derivative schemes (that $s A b r i n A . v 0$ solver usually relies on) were replaced with 15-point intrinsically optimized (IOFD) ones. On the same way, the 10-point standard FD filtering schemes usually employed were here replaced with 15-point ones, that is, of higher accuracy. One can here recall that, for not partly degrading the very high accuracy offered by the IOFD derivative schemes, it would have been ideally needed to make use of filtering schemes allotted with still wider stencil extent (19, or 21 points). Because of practical reasons, however, this option was not retained here.

Both CAA calculations were run for a total of 12,000 time steps (i.e. approx. $42 \%$ of the temporal extent of the CFD calculation), which corresponded to a physical duration of $60 \mathrm{~ms}$. The computations were processed in parallel over 480 cores, each calculation requiring a CPU time of 45 hours. Regarding the total number of grid points (66 millions) and iterations (12,000) involved, this represents an average CPU time of approx $98 \mu \mathrm{sec} / \mathrm{cell} /$ iteration (which a non negligible part was devoted to the I/O operations needed for reading the 101 Gigabytes of binary data that constituted the CFD-CAA source signal).

During each CAA calculation, the perturbed pressure field was stored every 2000 iterations, that is, at every one sixth of the total duration. The time history of all perturbed quantities was also stored in some specific points of the computational box, which corresponded to either microphone locations were experimental data had been recorded, or to numerical probes were CFD data had been acquired for analysis purposes. Those time signatures were processed by means of Fourier transforms, delivering associated frequency spectra (Power Spectral Density, PSD).

\section{CFD-CAA Coupled Calculations of the NLG: Baseline Configuration}

As was said, the first calculation corresponded to the baseline configuration, with a CAA computational set up that had been defined as close as possible to the CFD-FWH calculation one ('free-field' uniform steady mean flow). Such a calculation aimed at assessing further the present CFD-CAA coupling exercise, by direct comparison against other results of either numerical or experimental natures, which had been acquired and/or processed under the same conditions (e.g. homogeneous propagation medium).

\section{A. Calculations Results}

Figure 11 displays the instantaneous perturbed pressure field that was obtained at four different moments after the transient had evacuated the CAA computational box (which required approx. 4000 iterations, that is, one third of the calculation duration). As one can see, once injected within the computational domain via the weak coupling surface (cubic box, drawn here in purple), the CFD-CAA source signal was CAA-propagated up to the 
far field. One can notice that the resulting acoustic emission was somehow irregular, presenting an intermittent character, which is to be related to the one exhibited by the CFD source signal itself.

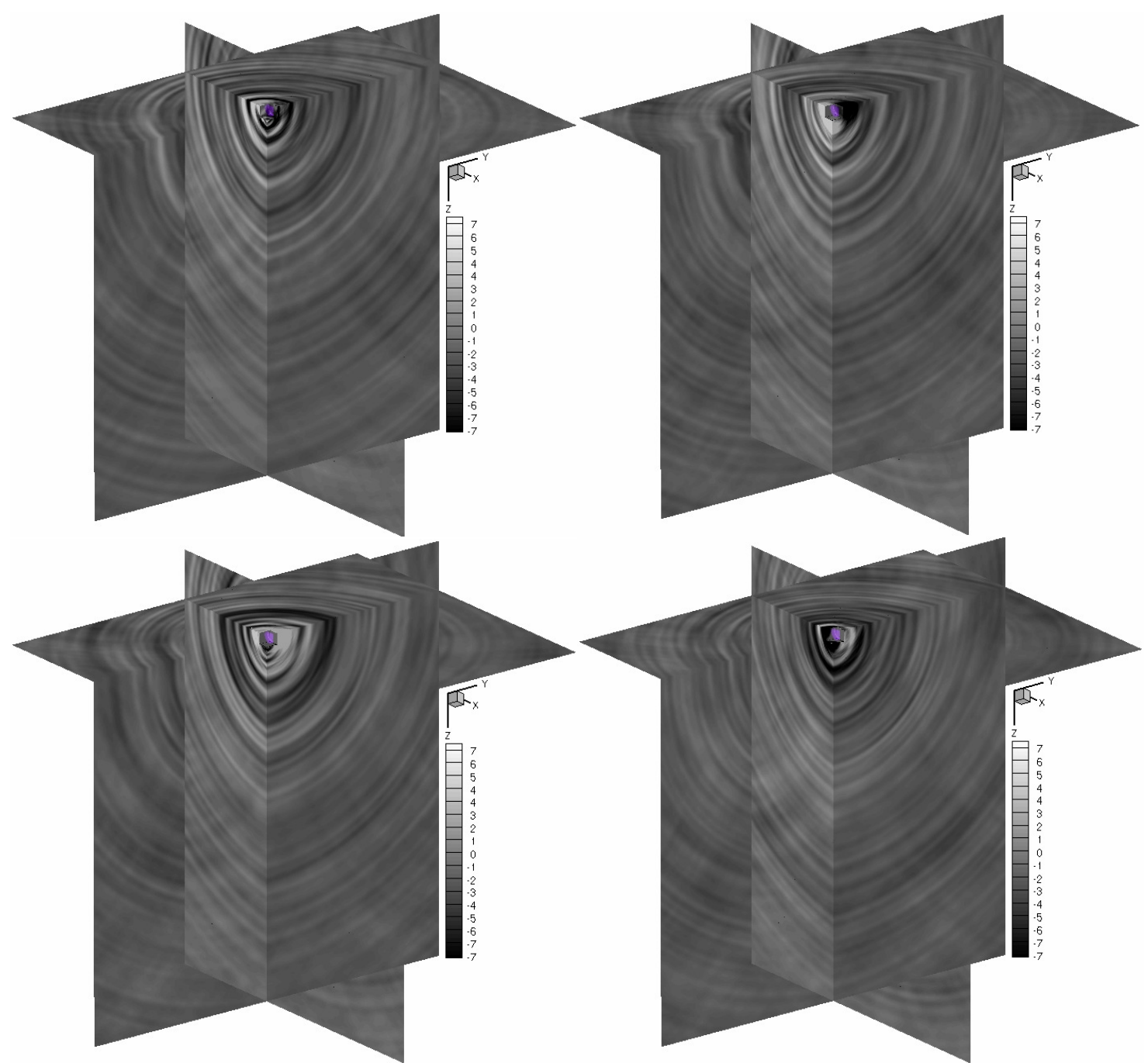

Figure 11. CFD-CAA Calculation of the NLG Baseline configuration ('free field' uniform mean flow). Clockwise, from top/left: instantaneous perturbed pressure field (in $P_{a}$ ) obtained at 3, 4, 5 and 6 sixth of the total duration (i.e. at iteration $6000,8000,10000$ and 12000, respectively)

One can here point out that the CAA calculation went out nicely, with no major numerical issue to be noticed. However, it is worth mentioning that two side effects were observed; first, it appeared that, although minor, some numerical reflections had occurred at the outer periphery of the domain. The latter may have been provoked and/or enhanced by some very low frequency $(<200 \mathrm{~Hz})$ contents that had visibly transited from the CFD source signal into the CAA computational box (see next section). Second, as was to be expected (see section IV.A.3), it was noticed that the coupling surface had been occasionally impinged by unsteady aerodynamic occurrences of rather high intensity. Although these incidents visibly remained low enough for not jeopardizing the calculation or corrupting too much the data, it is difficult to state on how far they impacted the final result.

\section{B. Validation, via Direct Comparison against Experimental and/or other Numerical Results}

With the view of tentatively validating the previous CFD-CAA coupled calculation and its underlying methodology, several comparison exercises were conducted. 
First, the efficiency of the coupling procedure and of its underlying NRI 2,8,16 technique was here checked again, by verifying that the CFD source signal was properly injected within the CAA domain; by looking at Figure 12, one can appreciate that the patterns of the prescribed source signal (which was plotted here over the coupling interface) were well recovered within the CAA domain. Here, however, it is important to notice that such a checking could validate the coupling step only, and not the entire procedure. Indeed, one can here recall that the CFD source signal
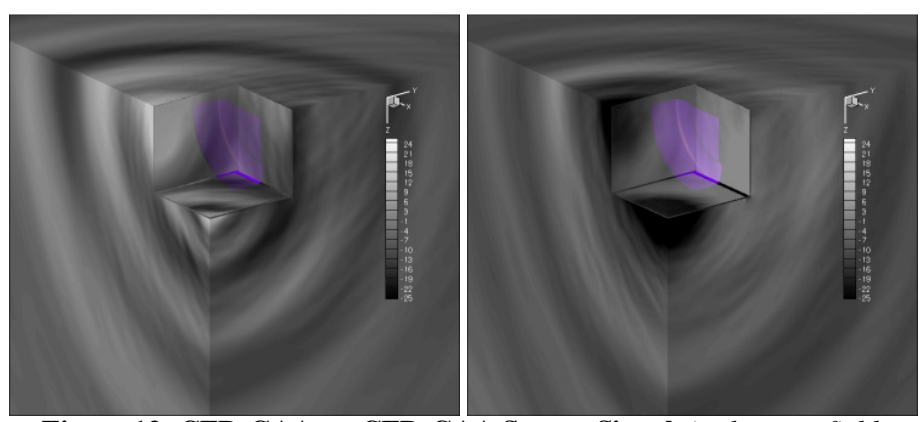

Figure 12: CFD-CAA vs. CFD-CAA Source Signal, in the near-field; CFD-CAA source signal, as prescribed over the coupling interface (cubic box) and propagated (slices). Instantaneous perturbed pressure fields obtained at the half (left) and the end (right) of the calculation, respectively

itself could have ever been corrupted before it was CAA-injected, because of spurious and/or aliased contents possibly inherited from the various manipulations ${ }^{14,28}$ it had been submitted to (e.g. the signal was successively CFD-interpolated in space, CFD-sampled in time, and CAA-sampled in space).

Therefore, in a second time, another validation exercise was conducted; the idea here was to compare directly the present CFD-CAA outputs with those that had been initially gathered during the CFD stage; Figure 13 compares the perturbed pressure fields that were delivered by both the CFD and the CFD-CAA calculations at the exact same moment, that is, at one sixth (2000 iterations) of the CAA simulation. Such comparison must be taken in a qualitative sense only, since the levels of the CFD perturbed field had to be adjusted, here; indeed, due to the drifting nature of the CFD calculation (see section IV.A.6), the associated overall stationary flow (which had been obtained by time averaging all the CFD realizations) was not suitable enough for allowing a proper derivation of CFD perturbed quantities oscillating around a same (null or constant) value. Thus, for comparison purpose, the CFD levels were here adjusted so that the acoustic waves of the near-field emerge more or less clearly from the continuum background. Despite of its sole indicative character, such a comparison exercise nevertheless reveals that both perturbed fields exhibited a similar dynamic, in terms of acoustic emission (to the obvious exception of that fraction coming from the NLG's leg, which was not accounted for in the CFD-CAA case - see section IV.A.1). This comparison also reveals that, while they rapidly vanished in the CFD stage, the acoustic waves propagated
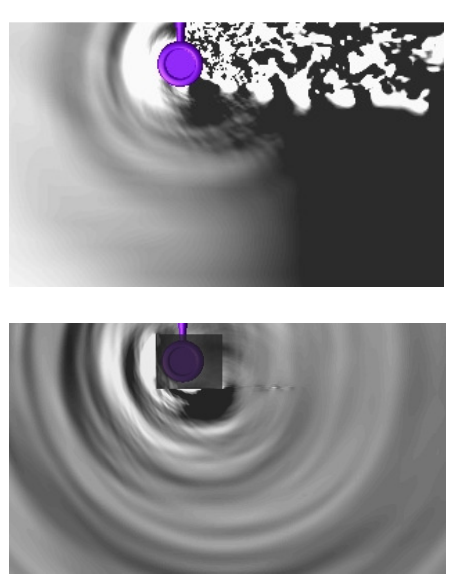

Figure 13. CFD-CAA vs. CFD, in the near-field; Instantaneous perturbed pressure, as CFD (top) and CFD-CAA (bottom) computed at $1 / 6^{\text {th }}$ of the calculation away in the CAA one, as was expected.

With the view of comparing both the CFD and the CFD-CAA perturbed fields still more closely, a third nearfield validation exercise was then attempted; this was made by comparing the time signatures that had been CFD- and CAA-recorded for six identical probes, which were located in the immediate vicinity of the coupling interface (see Fig. 14, bottom/right). First, from a qualitative point of view, one can see on left side of Figure 14 that each pair of time signatures exhibit very similar patterns, which match quite closely. This is more especially true for the probes located upstream (P1 to P4) or right under (P5) the landing gear axle, whatever their relative distance to the coupling surface is. For the probe located immediately downstream the gear (P6), however, the agreement between the two time signatures is less satisfactory, as patterns that do not always match closely. Although there is no sound explanation for such a qualitative mismatch, it may find its source in possible spurious effects ${ }^{2,5,8}$ coming from the sporadic impingement of strong unsteady aerodynamic occurrences onto the downstream part of the coupling interfaces, a thing that had been expected (see section IV.B.3) and observed (see section V.A) to occur.

Now, from a quantitative point of view, it appears that all time signatures coming from the CFD-CAA calculation generally exhibit higher amplitudes than those associated with the CFD simulation. Needless to say, part of such mismatch in amplitude has certainly to see with the fact that both calculation methods present very different dissipative features (especially considering that the CAA stage here relied on 15-points IOFD schemes, which present a much higher accuracy than the $2^{\text {nd }}$ order finite volume ones used for the CFD consumption).

For checking that point, the frequency spectra (PSD) associated with each time signature was determined (this being achieved with the help of windowed Fourier transforms, with a frequency accuracy of approx 100 $\mathrm{Hz}$ ); as one can see on right side of Fig. 14, part of the amplitude mismatch previously observed effectively finds its 
source in the lower accuracy (and thus higher dissipative character) of the CFD stage; indeed, by comparing the upper part (e.g. $f>3 \mathrm{kHz}$ ) of spectra recorded at probes $\mathrm{P} 4$ to $\mathrm{P} 1$, one can see how the higher frequencies appear to be less and less resolved by the CFD, as the distance between the probe and the gear increases. Although the same phenomena can also be observed on the CFD-CAA outputs, it happens at a much lower rate, visibly because of the higher accuracy features that the CAA stage relies on.
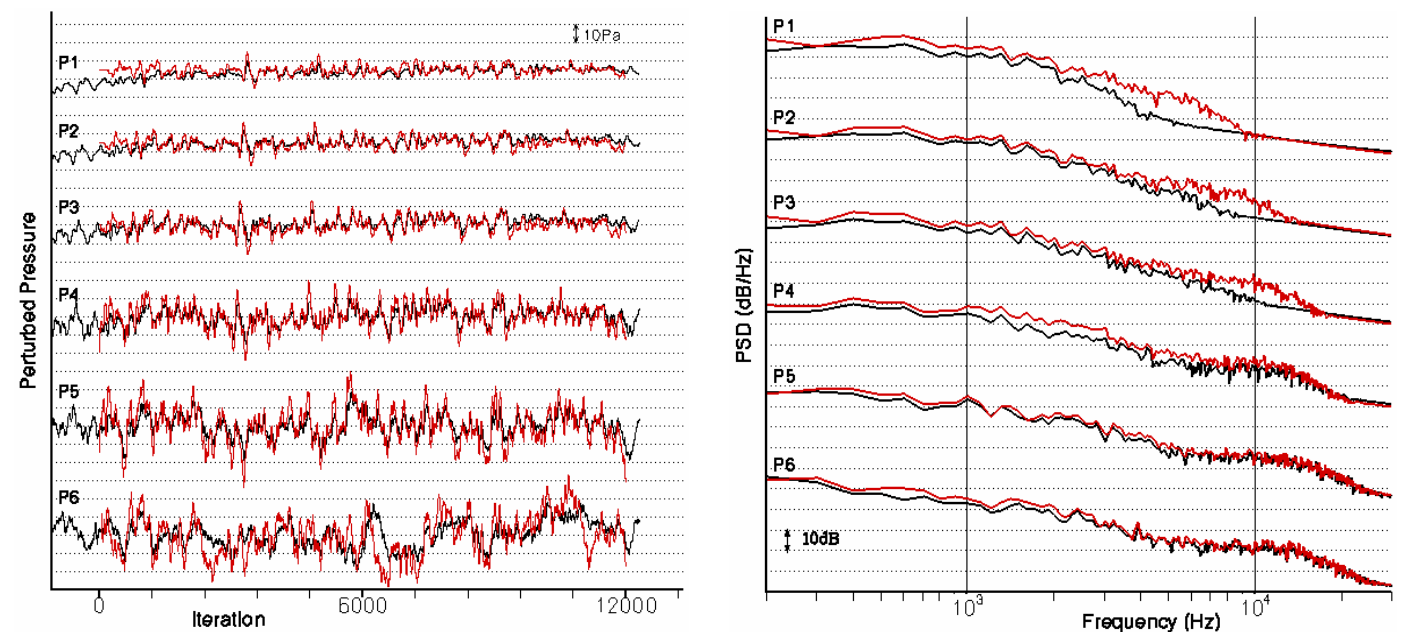

Figure 14. CFD-CAA vs CFD, in the very near-field. Perturbed pressure time signatures (top/left) and spectra (top/right), as $C F D$ (black) and CFD-CAA (red) recorded for 6 probes (right) during the entire CFD-CAA simulation
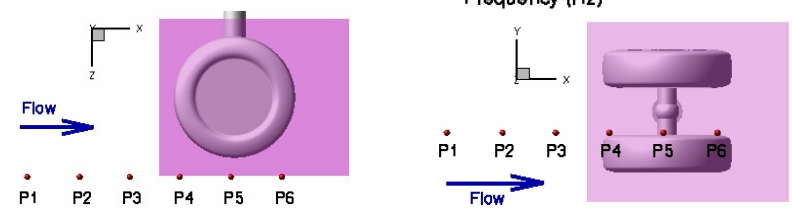

On another hand, by comparing now the lower part (e.g. $f<3 \mathrm{kHz}$ ) of all spectra, one can notice that an amplitude mismatch also occurs over the low- to mid- frequency range, and more particularly for the probes located further away from the coupling interface (P1 to P3). Although there is no sound explanation for such an amplitude mismatch, one can wonder again about possible spurious contents that could have been generated by the impingement of unsteady aerodynamic occurrences onto the CFD-CAA coupling interface, before to be CAA-propagated everywhere in the domain. One element that goes in the sense of such hypothesis is the fact that spectra excerpted from probes located further downstream the gear revealed that, over the wake region, the CFD signal exhibited much more important contents of either low or high frequency contents.

Apart from that, as said above, one can also wonder about any other spurious effect that could have impacted the CFD source signal before it was injected within the CAA domain, because of the various manipulations it was submitted to, once CFD-computed. For checking that point, it is planned to extend the present spectra analyses, by applying them also to the CFD-CAA source signal itself, in addition to the time signature resulting from the overall CFD-CAA coupling operation (that is, CFD signal manipulation, CFD-CAA forcing, CAA propagation, etc), as was done here.

\section{Mid-Field Validation; CFD-CAA against Experiments and CFD-FWH (solid surface)}

In a second time, the validation exercise was extended to the midfield region, where experimental data had been recorded at several locations, approx. $13 R$ away from the mock-up in the flyover direction (see Fig. 9 and left/top of Fig. 15). One can here remind that, since the experimental data had been corrected from the refraction effects by the open jet shear layers, they could be safely compared with the CFD-CAA results coming from the present (uniform mean flow) calculation case.

On that stage however, one can notice that there is a legitimate concern about possible bias that might have corrupted part of these mid-field experimental data, because of installation (e.g. reflection) effects that could have been induced onto the microphones by their mounting device (a rigid cross-like antenna, composed with two branches of approx $13 R$ length). Considering nevertheless the relatively small thickness and the tubular shape of each branch, such possible bias is expected to have played a significant role (if any) over the upper frequency range, rather than on the lower one - which was primarily targeted on, here.

On another hand, it is worth mentioning that, compared to the signal acquisition time used in the experiment $(20 \mathrm{sec})$, the CFD-CAA simulation time $(0.06 \mathrm{sec})$ was much shorter. In addition to that, because of the transient time needed for the first wave front to reach the probes location, the effective length of the useful signal that was numerically recorded was even shorter. As a consequence, one could expect the spectra analysis of the CFD- 
CAA outputs to be much less accurate than the one applied to the experimental data. More precisely, while the latter had been Fourier Transform (FT) processed with many averaging blocks and a maximal frequency step (that is, accuracy) of approx. $10 \mathrm{~Hz}$, the former where FT-handled with only a few averages, and a sampling frequency of no less then $100 \mathrm{~Hz}$.

Despite of all that, this mid-field validation exercise turned out to be rather satisfactory, as one can see on right side of Figure 15, which compares spectra coming from both the experiment (in black) and the CFD-CAA coupled calculation (in red). Note that, for indicative purpose, the spectra coming from CFD-FWH (solid surface) calculations that had been previously achieved for the same NLG configuration ${ }^{13}$ were also plotted here (in blue dashes).

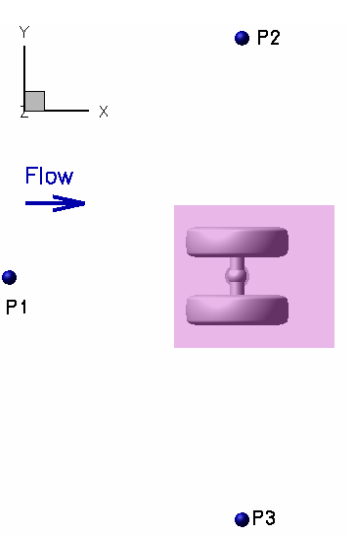

Figure 15. CFD-CAA vs. Experiments and CFD-FWH, in the mid-field. Right side: Perturbed pressure spectra, as recorded for 4 probes of the mid-field (top). Experiments in black, CFD-FWH (solid surface) in blue dashes, and CFD-CAA in red

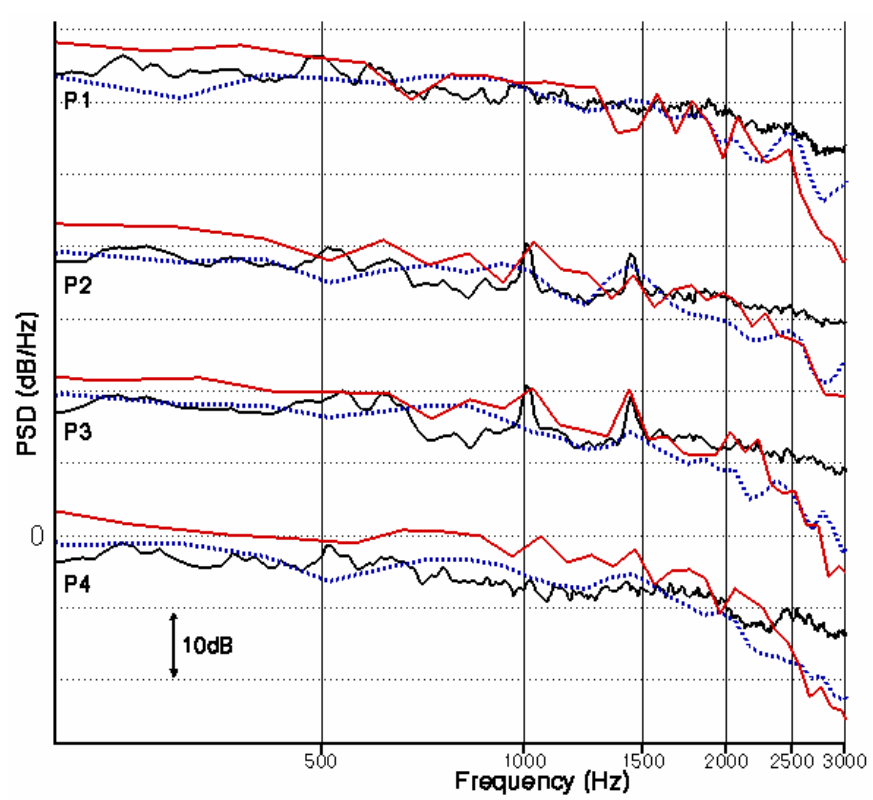

As one can see on such Fig. 15, CFD-CAA outputs reproduce fairly well the experimental ones, especially for the probes P1 to P3. In particular, the two tonal emissions that were experimentally recorded in the gear's lateral directions appear to have been correctly captured by the CFD-CAA calculation (and even better than by the CFD-FWH one, compare for instance the results obtained at $1 \mathrm{kHz}$ and $1.5 \mathrm{kHz}$, for probes $\mathrm{P} 2$ and $\mathrm{P} 3$ ).

On another hand, one can observe that the CFD-CAA amplitude levels are still overestimated by a few $\mathrm{dB}$ over the lower part of the frequency range. This is more visible on probe $\mathrm{P} 4$, where the mismatches occurring between the CFD-CAA and either the experimental or the CFD-FWH results is more visible. On that stage however, one can underline that, because this particular probe P4 was located downstream the NLG (see Fig 9 and top left of Fig 15), it may have suffered more importantly than the three others from possible bias of methodological / numerical origin; first, such P4 probe may have lacked from correct acoustic information, due to the fact that the (downstream) coupling interface \#2 had been suppressed intentionally, for stability reasons (see section IV.A.3). Second, it may also have been enhanced with incorrect acoustic information, because of the possible spurious effect coming from the impingement of high intensity aerodynamic occurrences onto the downstream part of the remaining coupling interfaces (especially the \#5).

Apart from that, one can notice how both the CFD-CAA and the CFD-FWH amplitude levels rapidly and similarly decay for frequencies higher than $2,5 \mathrm{kHz}$. Considering that the common denominator between both hybrid approaches is the CFD stage, one can infer that such effect is solely due to the dissipation by the CFD grid and schemes, which was to be expected.

\section{Far-Field Validation; CFD-CAA against Experiments and CFD-FWH (solid and porous surface)}

Finally, the previous validation attempt was repeated, but this time for the probes located in the far-field region (that is approx. $33 R$ to $40 R$ away from the mock-up, in the flyover direction - see left/top of Fig. 16). Results are displayed on Figure 16, which compares spectra coming from both the experiment (in black) and the hybrid calculations, whatever the latter were obtained following a CFD-CAA (in red), a CFD-FHW/solid surface (in blue dashes), or a CFD-FWH/porous surface (in blue lines) scenario.

Again, except for some amplitude mismatches occurring here and there (see below), the agreement between all results is fairly good, as both hybrid approaches reproduce quite correctly the general pattern of experimental 
records. This is rather satisfactory, especially if one considers that the spectra analysis of CFD-CAA (as well as CFD-FWH/porous) results was even less accurate in the present case than in the previous (mid-field) one, because of an increased transient time needed for the first wave front to reach the far-field region (a thing that reduced the useful signal length to $0.04 \mathrm{sec}$ ).

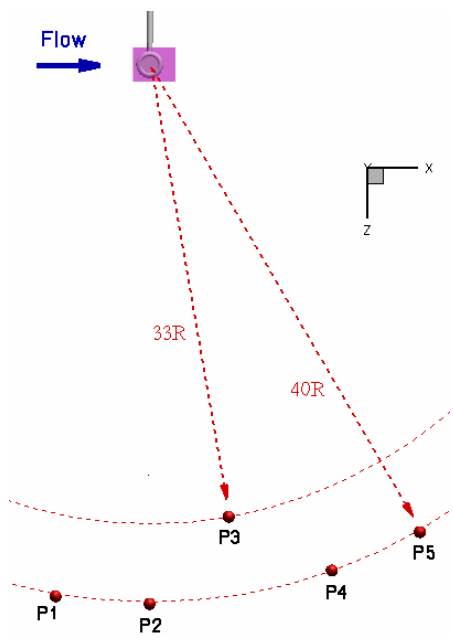

Figure 16. CFD-CAA vs. Experiments and CFD-FWH, in the far-field. Right side:

Perturbed pressure spectra, as recorded for 5 probes of the far-field (top). Experiments in black, CFD-FWH (solid and porous surfaces, in blue dashes and lines, respectively), $C F D$ CAA in red

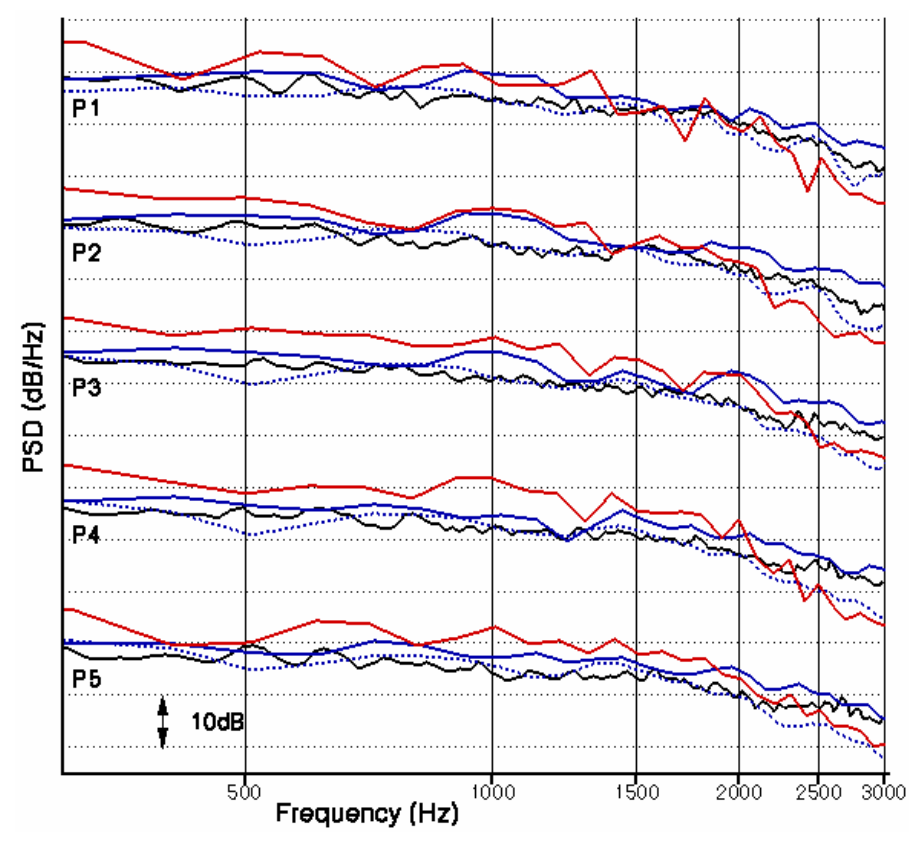

One can notice that, over the lower frequency range, CFD-CAA outputs generally exhibit higher levels than all other data, this being more especially visible on probes located downstream the NLG (P3 to P5). This last observation tends to confirm that the low frequency mismatch previously observed in the near- and mid-fields might effectively come from what happened on the downstream part of the coupling interfaces (e.g. the impingement by high intensity aerodynamic fluctuations). Here, however, such an excess of low frequency levels could also have been numerically aggravated by possible spurious reflections coming from the free-field boundary condition, which is known to be less efficient for low frequency contents exiting the domain peripheral border (which, here, was located quite close to the probes, see Fig. 9). On another hand, one can notice that, over the higher frequency range, the CFD-FWH/porous results generally exhibit higher amplitude than all other data, on the contrary to the CFD-CAA one, which follows quite closely the CFD-FWH/solid patterns.

Here, it is worth mentioning that, as shown in Ref. 8, cross-validating two numerical hybrid approaches is generally less obvious than it seems, as it encounters the difficulty of comparing calculation results coming not only from different methods (e.g. CAA vs. FWH), but also from different combinations of methods (e.g. CFDCAA and CFD-FWH), with possible mismatches inherited from either 1) the intrinsic modelling specificities of each methods or 2) the cumulative uncertainties inherited from their weak-coupling with a third one (e.g. unsteady CFD). In addition to that, if one considers again 1) the rather poor accuracy of spectral analyses to which all numerical data were submitted here, and 2) the fact that the experimental trends were globally captured by both hybrid methods, it appears that the present cross-validation exercise is rather convincing.

\section{CFD-CAA Coupled Calculations of the NLG: Alternative Configuration}

As was said previously, and following what had been done in Ref. 8, a second computation was conducted, so as to assess the sole mean flow effects that could have been induced by the facility jet on the experimental data. For doing so, the previous calculation was repeated, its free-field uniform mean flow being simply replaced with its facility-installed counterpart.

\section{A. Calculations Results and Early Analysis}

Figure 17 displays the instantaneous perturbed pressure field that was obtained either at one half or at the end of the calculation (iterations 6000 and 12000, respectively). When comparing those plots with their free-field counterparts of Figure 11 (first and last plots, respectively), one can observe some of the mean flow effects by the facility jet (see for instance what happens upstream the $x$-axis, where the jet exits the facility nozzle). 


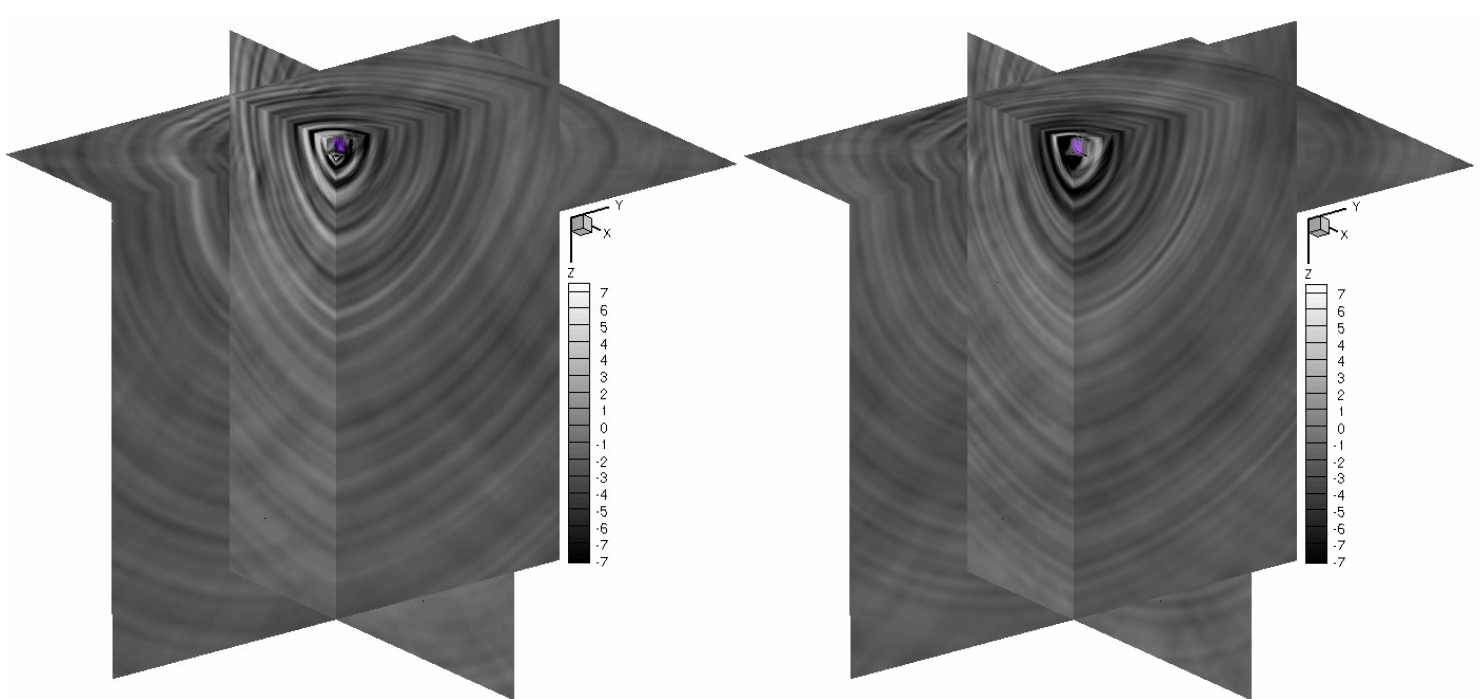

Figure 17. CFD-CAA Calculation of the NLG Alternative Configuration ('facility installed jet'). Instantaneous perturbed pressure field, as recorded (in $P_{a}$ ) at one half (left) and at the end (right) of the calculation

Those effects appear more clearly on Fig 18, which compares the present 'facility-installed jet' calculation result to the previous 'free field' ones, as they were recorded within the mid-field at the end of the calculation (iteration 120000). As one can see, compared to the homogeneous medium case, the acoustic waves are now modified as they cross the jet shear layers, and then propagate within a region where the flow is null. These cumulated refraction and (no) convection effects are more especially visible upstream the $x$-axis, where the shear layers are denser (see right side of Fig. 10).
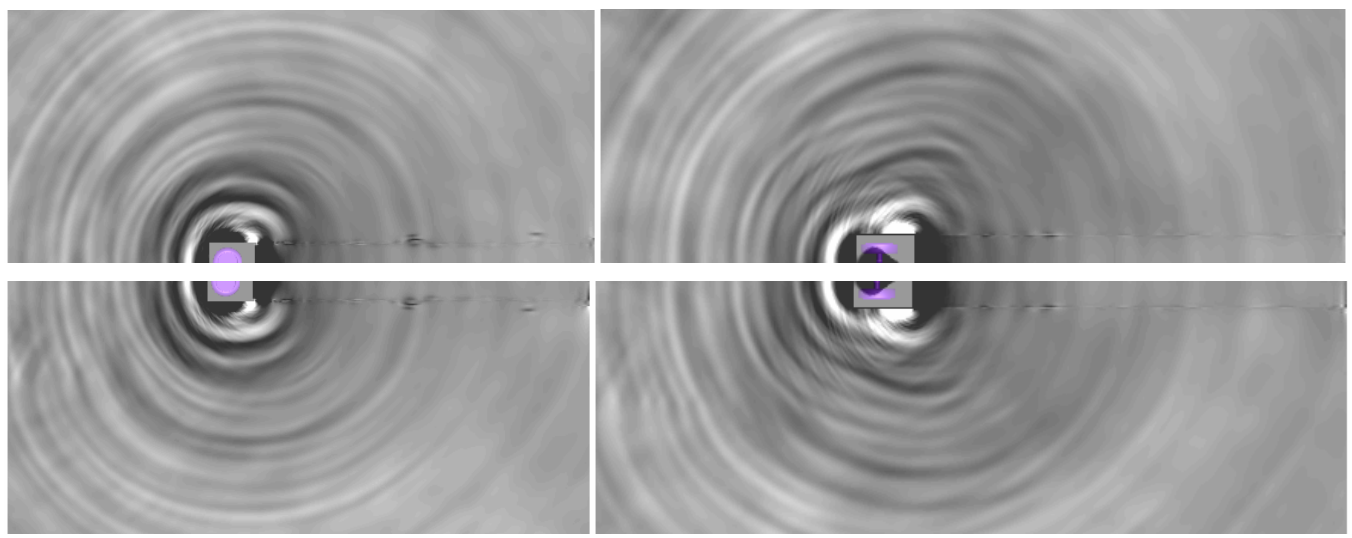

Figure 18. CFD-CAA Calculation of the NLG, baseline vs. alternative configurations. Mean flow effects associated with the 'facility installed' (bottom) NLG configuration, compared to the 'free field' one (top). Instantaneous perturbed pressure of both configurations, as recorded within the $y$ - (left) and z-planes (right) at the end of the calculation. For comparison purposes, the images associated with the 'free field' configuration (top) were mirrored, by symmetry

Finally, these effects by the facility-installed jet on the NLG acoustic emission are highlighted on Figure 19, which provides the differences occurring between both perturbed pressure fields at the end of the calculation. Here, one can clearly see how these mean flow effects impact almost all the regions located outside the jet, especially in some particular directions where they appear as being reinforced.

This point shall be investigated further in an immediate future; among other things, it is planned to conduct again the mid- and far- validation exercises presented at section $\mathrm{V}$, by comparing this time the numerical outputs coming from the present calculation against the experimental data that were left uncorrected from the mean flow effects. This shall allow numerically assessing the relevance of the refraction effect correction ${ }^{28}$ that is $^{2}$ commonly applied to acoustic data recorded in Onera's CEPRA 19 open jet anechoic wind tunnel. Here, it is worth mentioning that a purely CFD-FWH approach could not be able to provide such assessment, which shall contribute to a more meaningful comparison between experimental acoustic data and noise predictions based on a free-field configuration. Such an outcome could thus constitute an important step in employing the LAGooN measurements for assessing further acoustic post-processing techniques (e.g. the FWH approach) usually 
employed for landing gear noise studies ${ }^{20-22}$, as suggested in the BANC initiative ${ }^{23}$.
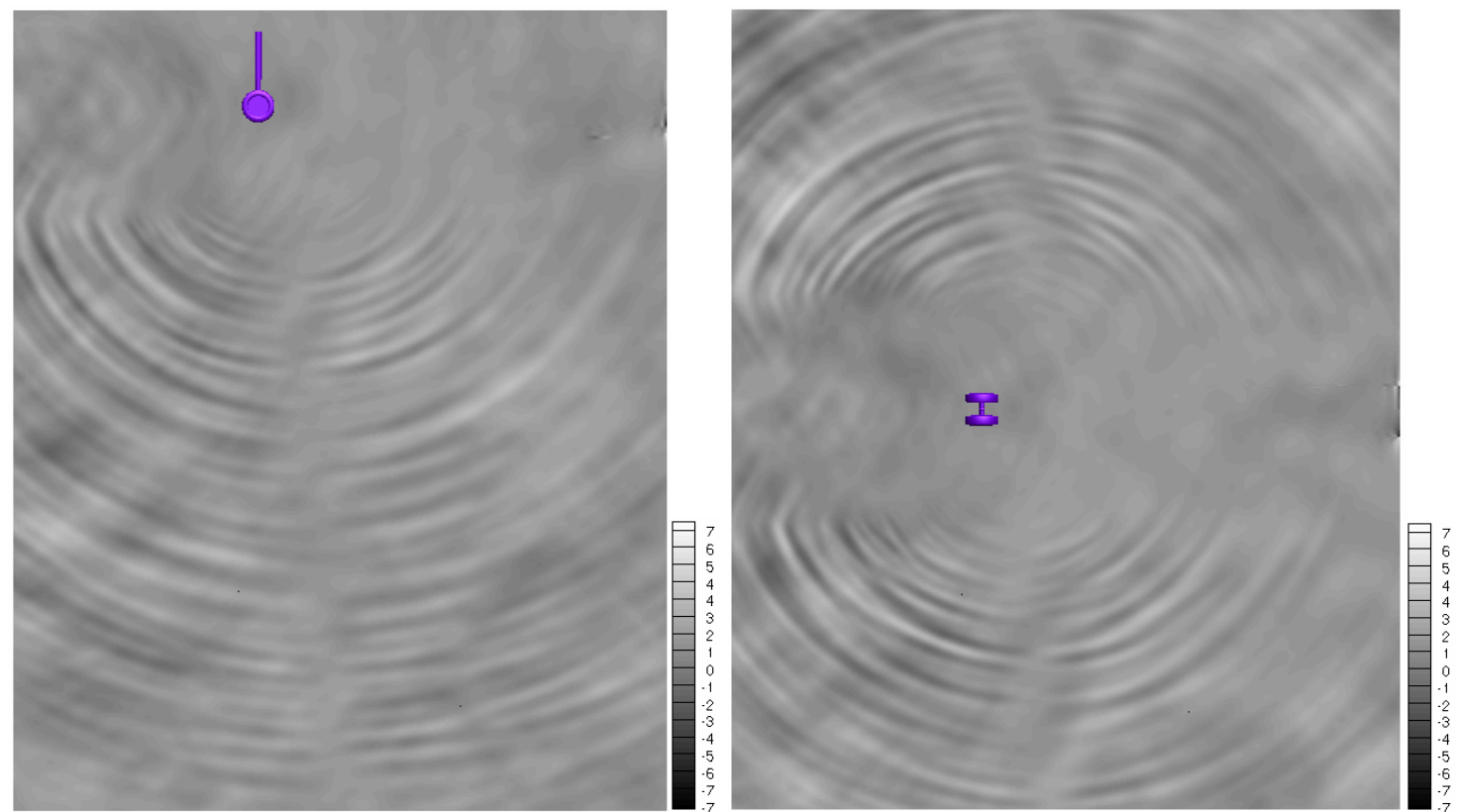

Figure 19. CFD-CAA Calculation of the NLG, baseline vs. alternative configurations. Mean flow effects associated with

the 'facility installed' NLG configuration, compared to the 'free field' one. Deltas between the instantaneous perturbed pressure field of both configurations, as recorded (in $P_{a}$ ) within the $y$ - (left) and $z$-planes (right) at the end of the calculation

\section{Conclusion}

The present work is part of a more extensive effort that aims at developing a robust and accurate CFD-CAA weak coupling approach, with respect to hybrid methods in aeroacoustics. In that regard, the so-called CFDCAA surface coupling procedure was here further assessed and validated through an application to a realistic problem, given by the aeroacoustics of a nose landing gear (NLG) in an approach flight.

First, the main methodological aspects underlying such an application of the CFD-CAA surface coupling procedure to an industrial-like problem of this nature were detailed and discussed, so as to serve as general guidelines. A first computation of the NGL configuration was then conducted, before it was compared against data coming from both experiments and alternative computations that had been previously devoted to the present NLG configuration. This allowed to validate further both the overall coupling methodology and its constitutive ingredients (NRI technique, IOFD schemes, etc.), as well as to assess better their effective strengths and potential weaknesses. Finally, a second calculation allowed investigating more phenomenological aspects, through the early assessment of some installation effects to which the NLG experiments could have been subjected to.

Although not fully completed yet, the present study delivers key insights about how the CFD-CAA surface weak coupling technique and its underlying hybrid approach can effectively be applied to real life problems, so as to help in improving the fidelity of numerical predictions. More insights shall be provided in a very near future, paving the way to more systematic utilization of such CFD-CAA surface coupling approach for solving realistic problems of aircraft noise.

\section{Acknowledgments}

Although the present work was funded by ONERA, it took direct benefit from the LAGooN project, which was supported and led by Airbus. The authors acknowledge Dr. Bastien Caruelle (Airbus) and Dr. Eric Manoha (Onera) for having granted them an access to the LAGooN experimental and numerical database. Authors also thank Mr. Laurent Sanders (Onera) for having provided them with insights and data coming from the CFD-FWH calculation campaign. Finally, authors acknowledge Dr. Mehdi Khorrami (NASA/LaRC), Dr. P. Spalart (Boeing), and Dr. A. Sengissen (Airbus) whose research works and/or informal exchanges helped in guiding the present study. 


\section{References}

${ }^{1}$ Ffowcs Williams, J. E. and Hawkings, D. L., "Sound generation by turbulence and surfaces in arbitrary motion," Philosophical Transactions of the Royal Society of London A, Vol. 342, 1969, pp. 264-321.

${ }^{2}$ Redonnet, S., "On the Numerical Prediction of Aerodynamic Noise via a Hybrid Approach - Part 1: CFD/CAA Surfacic Coupling Methodology, Revisited for the Prediction of Installed Airframe Noise Problem," AIAA Paper 2010-3709, 16th AIAA/CEAS Aeroacoustics Conference, Stockholm, Sweden, June 2010.

${ }^{3}$ Redonnet, S., "Simulation de la propagation acoustique en présence d'écoulements quelconques et de structures solides, par résolution numérique des équations d'Euler," PhD Thesis, Université Bordeaux I, 2001.

${ }^{4}$ Redonnet, S., Manoha, E. and Sagaut, P. "Numerical Simulation of Propagation of Small Perturbations interacting with Flows and Solid Bodies," AIAA Paper 2001-2223, 7th CEAS/AIAA Aeroacoustics Conference, Maastricht, The Netherlands, May 2001.

${ }^{5}$ Manoha, E., Herrero, C., Sagaut, P. and Redonnet, S., "Numerical Prediction of Airfoil Aerodynamic Noise," AIAA Paper 2002-2573, 8th CEAS/AIAA Aeroacoustics Conference, Breckenridge, USA, June 2002.

${ }^{6}$ Guenanff, R., "Couplage instationnaire Navier-Stokes/Euler pour la génération et le rayonnement des sources de bruit aérodynamique," PhD Thesis, Rennes University, 2004, No. 3138.

${ }^{7}$ Terracol, M., Manoha, E., Herrero, C., Labourasse, E., Redonnet, S. and Sagaut, P., "Hybrid Methods for Airframe Noise Numerical Prediction," Theoretical and Computational Fluid Dynamics, Vol. 19, No.3, July 2005.

${ }^{8}$ Redonnet, S., Lockard, D. P., Khorrami, M. R. and Choudhari, M. M., "CFD-CAA Coupled Calculations of a Tandem Cylinder Configuration to Assess Facility Installation Effects", AIAA Paper 2011-2841, 17th AIAA/CEAS Aeroacoustics Conference, Portland, USA, June 2011.

${ }^{9}$ Manoha E., Bulté J., Caruelle B., "LAGOON : an Experimental Database for the Validation of CFD/CAA Methods for Landing Gear Noise Prediction", AIAA 2008-2816, 14th AIAA/CEAS Aeroacoustics Conference, 5 - 7 May 2008, Vancouver, British Columbia, Canada

${ }^{10}$ Manoha E., Bulté J., Ciobaca V., Caruelle B., "LAGOON : further analysis of aerodynamic experiments and early aeroacoustics results", AIAA 2009-3277, 15th AIAA/CEAS Aeroacoustics Conference, 11 - 13 May 2009, Miami, Florida

${ }^{11}$ Ben Khelil S., Francois C., Deck S., "Zonal Detached-Eddy Simulation of the Flow Around Landing Gear Configuration", 20th AIAA Computational Fluid Dynamics Conference, 27-30 june 2011, Honolulu, Hawaii.

${ }^{12}$ Sanders L., Manoha E., Ben Khelil S., François C., "LAGOON : CFD/CAA coupling for Landing Gear Noise and Comparison with Experimental Database", 17th AIAA/CEAS Aeroacoustics Conference, Portland, USA, June 2011.

${ }^{13}$ Sanders L., Manoha E., Ben Khelil S., François C., "LAGOON : New Mach Landing Gear Noise Computation and further analysis of the CAA process", 18th AIAA/CEAS Aeroacoustics Conference, Colorado Springs, USA, June 2012.

${ }^{14}$ Cunha, G. and Redonnet, S., "On the Signal Degradation Induced by the Interpolation and the Sampling Rate Reduction in Aeroacoustics Hybrid Methods," International Journal for Numerical Methods in Fluids, June 2012.

${ }^{15}$ Cunha, G. and Redonnet, S., "Towards a Robust and Accurate CFD-CAA Coupling Procedure for Hybrid Methods in Aeroacoustics - Part 1: On the Optimization of CFD/CAA Coupled Calculations," AIAA paper 2012-2063, 18th AIAA/CEAS Aeroacoustics Conference, Colorado Springs, USA, June 2012.

${ }^{16}$ Redonnet, S. and Cunha, G., "Towards a Robust and Accurate CFD-CAA Coupling Procedure for Hybrid Methods in Aeroacoustics - Part 2: On the Application of the CFD-CAA Surface Weak Coupling Methodology to Realistic Aircraft Noise Problems," AIAA paper 2012-2191, 18th AIAA/CEAS Aeroacoustics Conference, Colorado Springs, USA, June 2012.

${ }^{17}$ Polacsek, C., Burguburu, S., Redonnet, S. and Terracol, M., "Numerical Simulations of Fan Interaction Noise using a Hybrid Approach," AIAA Journal, Vol. 44, No. 6, June 2006.

${ }^{18}$ Redonnet, S., Desquesnes, G., Manoha E. and Parzani, C., "Numerical Study of Acoustic Installation Effects with a CAA Method, " AIAA Journal, Vol 48 n5, May 2010.

${ }^{19}$ Redonnet, S. and Druon, Y., "Computational AeroAcoustics of Realistic Co-Axial Engines," AIAA Journal, Vol 50 n ${ }^{\circ}$, May 2012.

${ }^{20}$ Keating, A., Dethioux, P., Satti, R., Noelting, S., Louis, J., Van de Ven, T. and Vieito, R., "Computational Aeroacoustics Validation and Analysis of a Nose Landing Gear," AIAA Paper n²009-3154, 15th AIAA/CEAS Aeroacoustics Conference, Miami, USA, May 2009.

${ }^{21}$ Noelting, S., Bres, G. and Dethioux, P., "A Hybrid Lattice-Boltzmann/FW-H Method to Predict Sources and Propagation of Landing Gear Noise," AIAA Paper 2010-3976, 16th AIAA/CEAS Aeroacoustics Conference, Stockholm, Sweden, June 2010.

${ }^{22}$ Vuillot, F., Houssen, F., Manoha, E., Redonnet, S. and Jacob J., “, "Applications of the CEDRE unstructured flow solver to landing gear unsteady flow and noise predictions", AIAA Paper 2011-2944-393, 17th AIAA/CEAS Aeroacoustics Conference, Portland, USA, June 2011.

${ }^{23}$ Lockard D., "Summary of the Tandem Cylinder Solutions from the Benchmark problems for Airframe Noise Computations-I Workshop", AIAA 2011-353, 49th AIAA Aerospace Sciences Meeting, 4-7 January 2011, Orlando, Florida.

${ }^{24}$ Cunha, G. and Redonnet, S., "Low-Dispersion High-Order Explicit Finite-Difference Schemes for Computational Aeroacoustics", submitted to International Journal for Numerical Methods in Fluids, November 2012.

${ }^{25}$ S. K. Lele, "Compact finite difference schemes with spectral-like resolution", Journal of Computational Physics 103 (1) (1992) $16-42$.

${ }^{26}$ C. K. W. Tam, J. C. Webb, "Dispersion-relation-preserving finite difference schemes for computational acoustics", Journal of Computational Physics 107 (8) (1993) 262-281.

${ }^{27}$ Cunha, G., "Optimization of a Aeroacoustics Hybrid Approach Based on a Weak Coupling of Unsteady Aerodynamic and Acoustic Propagation Methods", PhD Thesis, Toulouse University, 2012.

${ }^{28}$ Amiet R. K., "Correction of open jet wind tunnel measurements for shear layer refraction," AIAA paper 75-532, 2nd AIAA Aeroacoustics Conference, Hampton, 1975. 